\title{
CUTTING A PART FROM MANY MEASURES
}

\author{
PAVLE V. M. BLAGOJEVIĆ ${ }^{1,2}$, NEVENA PALIĆ ${ }^{1}$, \\ PABLO SOBERÓN ${ }^{\circledR 3}$ and GÜNTER M. ZIEGLER ${ }^{\circledR} 1$ \\ ${ }^{1}$ Institut für Mathematik, FU Berlin, Arnimallee 2, 14195 Berlin, Germany; \\ email: blagojevic@math.fu-berlin.de,nevenapalic@gmail.com, ziegler@math.fu-berlin.de \\ ${ }^{2}$ Mathematical Institute SASA, Knez Mihailova 36, 11000 Beograd, Serbia \\ ${ }^{3}$ Baruch College, City University of New York, One Bernard Baruch Way, \\ New York, NY 10010, USA; \\ email: pablo.soberon-bravo@baruch.cuny.edu
}

Received 18 January 2018; accepted 26 August 2019

\begin{abstract}
Holmsen, Kynčl and Valculescu recently conjectured that if a finite set $X$ with $\ell n$ points in $\mathbb{R}^{d}$ that is colored by $m$ different colors can be partitioned into $n$ subsets of $\ell$ points each, such that each subset contains points of at least $d$ different colors, then there exists such a partition of $X$ with the additional property that the convex hulls of the $n$ subsets are pairwise disjoint.

We prove a continuous analogue of this conjecture, generalized so that each subset contains points of at least $c$ different colors, where we also allow $c$ to be greater than $d$. Furthermore, we give lower bounds on the fraction of the points each of the subsets contains from $c$ different colors. For example, when $n \geqslant 2, d \geqslant 2, c \geqslant d$ with $m \geqslant n(c-d)+d$ are integers, and $\mu_{1}, \ldots, \mu_{m}$ are $m$ positive finite absolutely continuous measures on $\mathbb{R}^{d}$, we prove that there exists a partition of $\mathbb{R}^{d}$ into $n$ convex pieces which equiparts the measures $\mu_{1}, \ldots, \mu_{d-1}$, and in addition every piece of the partition has positive measure with respect to at least $c$ of the measures $\mu_{1}, \ldots, \mu_{m}$.
\end{abstract}

2010 Mathematics Subject Classification: 52C35, 51M20 (primary); 55R20, 55N25 (secondary)

\section{Introduction and statement of the main results}

The classical measure partition problems ask whether, for a given collection of measures of some Euclidean space, the ambient Euclidean space can be partitioned in a prescribed way so that each of the given measures gets cut into equal pieces.

(c) The Author(s) 2019. This is an Open Access article, distributed under the terms of the Creative Commons Attribution licence (http://creativecommons.org/licenses/by/4.0/), which permits unrestricted re-use, distribution, and reproduction in any medium, provided the original work is properly cited. 
The first example of such a result is the well-known ham-sandwich theorem, conjectured by Steinhaus and later proved by Banach. It claims that given $d$ measures in $\mathbb{R}^{d}$, one can cut $\mathbb{R}^{d}$ by an affine hyperplane into two pieces so that each of the measures is cut into halves. Motivated by the ham-sandwich theorem, Grünbaum posed a more general hyperplane measure partition problem in 1960 [9, Section $4(\mathrm{v})]$. He asked whether any given measure in the Euclidean space $\mathbb{R}^{d}$ can be cut by $k$ affine hyperplanes into $2^{k}$ equal pieces. An even more general problem was proposed and considered by Hadwiger [10] and Ramos [15]: determine the minimal dimension $d$ such that for every collection of $j$ measures on $\mathbb{R}^{d}$ there exists an arrangement of $k$ affine hyperplanes in $\mathbb{R}^{d}$ that cut all measures into $2^{k}$ equal pieces. For a survey on the Grünbaum-Hadwiger-Ramos hyperplane measure partition problem, consult [3].

Furthermore, in 2001 Bárány and Matoušek [2] considered partitions of measures on the sphere $S^{2}$ by fans with the requirement that each angle of the fan contains a prescribed proportion of every measure.

In this paper, motivated by a conjecture of Holmsen, Kynčl \& Valculescu [12, Conjecture 3], we consider many measures in a Euclidean space, and instead of searching for equiparting convex partitions we look for convex partitions that in each piece capture a positive amount from a (large) prescribed number of the given measures.

DEFINITION 1. Let $d \geqslant 1$ and $n \geqslant 1$ be integers. An ordered collection of closed subsets $\left(C_{1}, \ldots, C_{n}\right)$ of $\mathbb{R}^{d}$ is called a partition of $\mathbb{R}^{d}$ if

(1) $\bigcup_{i=1}^{n} C_{i}=\mathbb{R}^{d}$

(2) $\operatorname{int}\left(C_{i}\right) \neq \emptyset$ for every $1 \leqslant i \leqslant n$, and

(3) $\operatorname{int}\left(C_{i}\right) \cap \operatorname{int}\left(C_{j}\right)=\emptyset$ for all $1 \leqslant i<j \leqslant n$.

A partition $\left(C_{1}, \ldots, C_{n}\right)$ is called convex if all subsets $C_{1}, \ldots, C_{n}$ are convex.

Let $m \geqslant 1, n \geqslant 1, c \geqslant 1$ and $d \geqslant 1$ be integers, and let $\mathcal{M}=\left(\mu_{1}, \ldots, \mu_{m}\right)$ be a collection of $m$ finite absolutely continuous measures in $\mathbb{R}^{d}$. Moreover, assume that $\mu_{j}\left(\mathbb{R}^{d}\right)>0$, for every $1 \leqslant j \leqslant m$. For us a measure is an absolutely continuous measure if it is absolutely continuous with respect to the standard Lebesgue measure.

We are interested in the existence of a convex partition $\left(C_{1}, \ldots, C_{n}\right)$ of $\mathbb{R}^{d}$ with the property that each set $C_{i}$ contains a positive amount of at least $c$ of the measures, that is

$$
\#\left\{j: 1 \leqslant j \leqslant m, \mu_{j}\left(C_{i}\right)>0\right\} \geqslant c
$$


for every $1 \leqslant i \leqslant n$. In the case when the measures are given by finite point sets, we say that a point set $X \subseteq \mathbb{R}^{d}$ is in general position if no $d+1$ points from $X$ lie in an affine hyperplane in $\mathbb{R}^{d}$. For the point set measures in general position Holmsen, Kynčl and Valculescu proposed the following natural conjecture [12, Conjecture 3].

Conjecture 1 (Holmsen, Kynčl, Valculescu, 2017). Let $d \geqslant 2, \ell \geqslant 2, m \geqslant 2$ and $n \geqslant 1$ be integers with $m \geqslant d$ and $\ell \geqslant d$. Consider a set $X \subseteq \mathbb{R}^{d}$ of $\ell n$ points in general position that is colored with at least $m$ different colors. If there exists a partition of the set $X$ into $n$ subsets of size $\ell$ such that each subset contains points colored by at least $d$ colors, then there exists such a partition of $X$ that in addition has the property that the convex hulls of the $n$ subsets are pairwise disjoint.

The conjecture was settled for $d=2$ in the same paper by Holmsen, Kynčl and Valculescu [12]. On the other hand, if instead of finite collections of points one considers finite positive absolutely continuous measures in $\mathbb{R}^{d}$, Soberón [16] gave a positive answer on splitting $d$ measures in $\mathbb{R}^{d}$ into convex pieces such that each piece has positive measure with respect to each of the measures. Moreover, he proved the existence of convex partitions that equipart all measures. A discretization of Soberón's result by Blagojević et al. [5] gave a positive answer to Conjecture 1 in the case when $m=d$. In addition, they were able to show that the set $X$ can be partitioned into $n$ subsets in such a way that all color classes are equipartitioned simultaneously.

In this paper we prove several continuous results of a similar flavor, trying to come closer to a positive answer to Conjecture 1 in the case when $m \geqslant d$. The first of the three results is the following.

THEOREM 2. Let $d \geqslant 2, m \geqslant 2, n \geqslant 2$, and $c \geqslant d$ be integers. If

$$
m \geqslant n(c-d)+d,
$$

then for every collection $\mathcal{M}=\left(\mu_{1}, \ldots, \mu_{m}\right)$ of $m$ positive finite absolutely continuous measures on $\mathbb{R}^{d}$, there exists a partition of $\mathbb{R}^{d}$ into $n$ convex subsets $\left(C_{1}, \ldots, C_{n}\right)$, which is a convex $n$-fan, such that each of the subsets has positive measure with respect to at least $c$ of the measures $\mu_{1}, \ldots, \mu_{m}$. In other words,

$$
\#\left\{j: 1 \leqslant j \leqslant m, \mu_{j}\left(C_{i}\right)>0\right\} \geqslant c
$$

for every $1 \leqslant i \leqslant n$.

The following two theorems have stronger statements-in Theorem 3 we additionally show that one of the measures can be equipartitioned without 
changing the bound on $m$, and in Theorem 4 we prove that the sum of all the measures can be equipartitioned if we allow the number of measures $m$ to increase.

THEOREM 3. Let $d \geqslant 2, m \geqslant 2$, and $c \geqslant d$ be integers, and let $n=p^{k}$ be a prime power. If

$$
m \geqslant n(c-d)+\frac{d n}{p}-\frac{n}{p}+1,
$$

then for every collection $\mathcal{M}=\left(\mu_{1}, \ldots, \mu_{m}\right)$ of $m$ positive finite absolutely continuous measures on $\mathbb{R}^{d}$, there exists a partition of $\mathbb{R}^{d}$ into $n$ convex subsets $\left(C_{1}, \ldots, C_{n}\right)$ that equiparts the measure $\mu_{m}$ with the additional property that each of the subsets has positive measure with respect to at least $c$ of the measures $\mu_{1}$, $\ldots, \mu_{m}$. In other words,

$$
\mu_{m}\left(C_{1}\right)=\cdots=\mu_{m}\left(C_{n}\right)=\frac{1}{n} \mu_{m}\left(\mathbb{R}^{d}\right)
$$

and

$$
\#\left\{j: 1 \leqslant j \leqslant m, \mu_{j}\left(C_{i}\right)>0\right\} \geqslant c
$$

for every $1 \leqslant i \leqslant n$.

THEOREM 4. Let $d \geqslant 2, m \geqslant 2$, and $c \geqslant d$ be integers, and let $n=p^{k}$ be a prime power. If

(a) $n(c-1) \geqslant m$ and $\max \{m, n\} \geqslant n(c-d)+(d n / p)-(n / p)+n$, or

(b) $n(c-1)<m$,

then for every collection $\mathcal{M}=\left(\mu_{1}, \ldots, \mu_{m}\right)$ of $m$ positive finite absolutely continuous measures on $\mathbb{R}^{d}$, there exists a partition of $\mathbb{R}^{d}$ into $n$ convex subsets $\left(C_{1}, \ldots, C_{n}\right)$ that equiparts the sum of the measures $\mu=\mu_{1}+\cdots+\mu_{m}$ with the additional property that each of the subsets has positive measure with respect to at least $c$ of the measures $\mu_{1}, \ldots, \mu_{m}$. In other words,

$$
\mu\left(C_{1}\right)=\cdots=\mu\left(C_{n}\right)=\frac{1}{n} \mu\left(\mathbb{R}^{d}\right)
$$

and

$$
\#\left\{j: 1 \leqslant j \leqslant m, \mu_{j}\left(C_{i}\right)>0\right\} \geqslant c
$$

for every $1 \leqslant i \leqslant n$. 
Previous solutions for measure partition problems relied on a variety of advanced methods from equivariant topology. Different configuration space/test map schemes (CS/TM schemes) related partition problems with the questions of non-existence of appropriately constructed equivariant maps from configuration spaces into suitable test spaces. For example, in the proof of the ham-sandwich theorem a sphere with the antipodal action appears as a test space. The test space in the Grünbaum-Hadwiger-Ramos hyperplane measure partition problem is again a sphere, but with an action of the sign permutation group, while the test space in the Bárány and Matoušek fan partition problem is a complement of an arrangement of linear subspaces equipped with an action of the Dihedral or generalized quaternion group. In this paper the proof of Theorem 2 is elementary and it does not use any topology. However, the proofs of Theorems 3 and 4 rely on a novel CS/TM scheme presented in Theorems 10 and 11: for the first time the test space is the union of an arrangement of affine subspaces, equipped in this case with an action of a symmetric group.

Furthermore, even stronger measure partition result can be obtained directly without any use of advanced methods of equivariant topology. We prove the following result with two similar arguments given in Sections 4.3 and 4.5.

THEOREM 5. Let $d \geqslant 2, m \geqslant 2, n \geqslant 2$, and $c \geqslant d$ be integers. If

$$
m=n(c-d)+d,
$$

then for every collection $\mathcal{M}=\left(\mu_{1}, \ldots, \mu_{m}\right)$ of $m$ positive finite absolutely continuous measures on $\mathbb{R}^{d}$, there exists a partition of $\mathbb{R}^{d}$ into $n$ convex subsets $\left(C_{1}, \ldots, C_{n}\right)$ that equiparts the first $d-1$ measures $\mu_{1}, \ldots, \mu_{d-1}$ with the additional property that each of the subsets has positive measure with respect to at least $c$ of the measures $\mu_{1}, \ldots, \mu_{m}$. In other words,

$$
\mu_{k}\left(C_{1}\right)=\cdots=\mu_{k}\left(C_{n}\right)=\frac{1}{n} \mu_{k}\left(\mathbb{R}^{d}\right)
$$

for every $1 \leqslant k \leqslant d-1$, and

$$
\#\left\{j: 1 \leqslant j \leqslant m, \mu_{j}\left(C_{i}\right)>0\right\} \geqslant c
$$

for every $1 \leqslant i \leqslant n$.

As a direct corollary of the proof of Theorem 5 given in Section 4.5 we get the following straightening.

COROLlaRy 6. Let $d \geqslant 2, m \geqslant 2, n \geqslant 2$, and $c \geqslant d$ be integers. If

$$
m=n(c-d)+d,
$$


then for every collection $\mathcal{M}=\left(\mu_{1}, \ldots, \mu_{m}\right)$ of $m$ positive finite absolutely continuous measures on $\mathbb{R}^{d}$ with the property that

$$
\mu_{d}\left(\mathbb{R}^{d}\right)=\mu_{d+1}\left(\mathbb{R}^{d}\right)=\cdots=\mu_{m}\left(\mathbb{R}^{d}\right)
$$

there exists a partition of $\mathbb{R}^{d}$ into $n$ convex subsets $\left(C_{1}, \ldots, C_{n}\right)$ that equiparts the measures

$$
\mu_{1}, \ldots, \mu_{d-1}, \mu_{d}+\cdots+\mu_{m}, \mu_{1}+\cdots+\mu_{m},
$$

and has the additional property that each of the subsets has positive measure with respect to at least $c$ of the measures $\mu_{1}, \ldots, \mu_{m}$. In other words,

$$
\#\left\{j: 1 \leqslant j \leqslant m, \mu_{j}\left(C_{i}\right)>0\right\} \geqslant c
$$

for every $1 \leqslant i \leqslant n$.

For Theorems 2, 3, 4 and 5 one can wonder: Are the lower bounds on the number of the measures $m$ optimal? We show that in the case when we require equipartition of $d-1$, out of $m$, measures the lower bound $m=n(c-d)+d$ from Theorem 5 is optimal.

THEOREM 7. Let $d \geqslant 1, n \geqslant 2$ and $c \geqslant d$ be integers, and let $m=n(c-d)+d-1$. There exists a collection of $m$ positive finite absolutely continuous measures in $\mathbb{R}^{d}$ such that for every partition of $\mathbb{R}^{d}$ into $n$ convex subsets $\left(C_{1}, \ldots, C_{n}\right)$ that equipart the first $d-1$ measures there is at least one part of the partition that has positive measure with respect to at most $c-1$ of the measures $\mu_{1}, \ldots, \mu_{m}$.

The technique used in the proof of Theorem 5 can be further utilized to determine the fraction of the measures obtained by a partition. We prove the following extension of Theorem 5 .

THEOREM 8. Let $d \geqslant 2, m \geqslant 2, n \geqslant 2$, and $c \geqslant d$ be integers. If

$$
m=n(c-d)+d,
$$

then for every collection $\mathcal{M}=\left(\mu_{1}, \ldots, \mu_{m}\right)$ of $m$ positive finite absolutely continuous measures on $\mathbb{R}^{d}$, there exists a partition of $\mathbb{R}^{d}$ into $n$ convex subsets $\left(C_{1}, \ldots, C_{n}\right)$ such that each of the subsets $C_{1}, \ldots, C_{n}$ has at least an

$$
\varepsilon=\frac{1}{n((n-1)(\lceil c / d\rceil-1)+1)} \geqslant \frac{d}{c n^{2}}
$$


fraction of at least $c$ of the measures $\mu_{1}, \ldots, \mu_{m}$. In other words,

$$
\#\left\{j: 1 \leqslant j \leqslant m, \mu_{j}\left(C_{i}\right) \geqslant \varepsilon \mu_{j}\left(\mathbb{R}^{d}\right)\right\} \geqslant c
$$

for every $1 \leqslant i \leqslant n$.

Furthermore, we prove that in some situations a fraction of measure each convex piece of a partition contains can be prescribed in advance. First, observe that if all the measures are equal we cannot hope to get more than a fraction of $1 / n$ in many measures for each convex piece, (the smallest part prohibits this). Nevertheless, we prove that we can get as close as $\alpha=1 / n$ as we want, provided we pay the price of using more measures.

THEOREM 9. Let $d \geqslant 2, m \geqslant 2, n \geqslant 2$, and $c \geqslant 2 d$ be integers, and let $0<\alpha<$ $1 / n$ be a real number. If

$$
m \geqslant(c-d)\left(\frac{1-\alpha}{1 / n-\alpha}\right)+d-1
$$

then for every collection $\mathcal{M}=\left(\mu_{1}, \ldots, \mu_{m}\right)$ of $m$ positive finite absolutely continuous measures on $\mathbb{R}^{d}$, there exists a partition of $\mathbb{R}^{d}$ into $n$ convex subsets $\left(C_{1}, \ldots, C_{n}\right)$ such that each of the subsets $C_{1}, \ldots, C_{n}$ has at least $\alpha$ fraction in at least $c$ of the measures $\mu_{1}, \ldots, \mu_{m}$. In other words,

$$
\#\left\{j: 1 \leqslant j \leqslant m, \mu_{j}\left(C_{i}\right) \geqslant \alpha \mu_{j}\left(\mathbb{R}^{d}\right)\right\} \geqslant c
$$

for every $1 \leqslant i \leqslant n$. Moreover, if $(1-\alpha) /((1 / n)-\alpha)$ is an integer, then it suffices to have

$$
m \geqslant(c-d)\left(\frac{1-\alpha}{1 / n-\alpha}\right)
$$

measures.

The reader may notice that, since $(1-\alpha) /((1 / n)-\alpha)>n$ if $\alpha>0$, if we make $\alpha \rightarrow 0$ we recover the same number of measures as Theorems 2 and 5 for $c \geqslant 2 d$. In Theorem 2 we have a stronger control on the kind of partitions obtained and in Theorem 5 we can also guarantee the equipartition of $d-1$ measures.

The rest of the paper is organized as follows. The proofs of Theorems 3 and 4 run in parallel and follow CS/TM schemes that are given in Section 2. The topological results about nonexistence of equivariant maps are proved in Section 3. Finally, the proofs of Theorems 2, 3, 4, 5, 7, 8 and 9 are given in Section 4. Furthermore, the proofs of Theorems 2, 5, 7, 8, and 9 can be read independently of the previous sections. 


\section{Existence of a partition from nonexistence of a map}

In this section we develop CS/TM schemes that relate the existence of convex partitions from Theorems 3 and 4 with the nonexistence of particular equivariant maps. These two CS/TM schemes are very similar to each other.

\subsection{Existence of an equipartition of one measure from nonexistence of a} map. Let $d \geqslant 2, m \geqslant 2, n \geqslant 1$, and $c \geqslant 2$ be integers, and let $\mathcal{M}=\left(\mu_{1}, \ldots\right.$, $\mu_{m}$ ) be a collection of finite absolutely continuous measures on $\mathbb{R}^{d}$. Throughout the paper we assume that $m \geqslant c$, since it is a requirement that naturally comes from the mass partition problem. Following notation from [6], let $\operatorname{EMP}\left(\mu_{m}, n\right)$ denote the space of all convex partitions of $\mathbb{R}^{d}$ into $n$ convex pieces $\left(C_{1}, \ldots, C_{n}\right)$ that equipart the measure $\mu_{m}$, as studied in [14], that is

$$
\mu_{m}\left(C_{1}\right)=\cdots=\mu_{m}\left(C_{n}\right)=\frac{1}{n} \mu_{m}\left(\mathbb{R}^{d}\right) .
$$

Now define a continuous map $f_{\mathcal{M}}: \operatorname{EMP}\left(\mu_{m}, n\right) \longrightarrow \mathbb{R}^{(m-1) \times n} \cong\left(\mathbb{R}^{m-1}\right)^{n}$ as

$$
\left(C_{1}, \ldots, C_{n}\right) \longmapsto\left(\begin{array}{cccc}
\mu_{1}\left(C_{1}\right) & \mu_{1}\left(C_{2}\right) & \ldots & \mu_{1}\left(C_{n}\right) \\
\mu_{2}\left(C_{1}\right) & \mu_{2}\left(C_{2}\right) & \ldots & \mu_{2}\left(C_{n}\right) \\
\vdots & \vdots & \ddots & \vdots \\
\mu_{m-1}\left(C_{1}\right) & \mu_{m-1}\left(C_{2}\right) & \ldots & \mu_{m-1}\left(C_{n}\right)
\end{array}\right) .
$$

The symmetric group $\mathfrak{S}_{n}$ acts on $\operatorname{EMP}\left(\mu_{m}, n\right)$ and $\left(\mathbb{R}^{m-1}\right)^{n}$ as follows

$$
\pi \cdot\left(C_{1}, \ldots, C_{n}\right)=\left(C_{\pi(1)}, \ldots, C_{\pi(n)}\right)
$$

and

$$
\pi \cdot\left(Y_{1}, \ldots, Y_{n}\right)=\left(Y_{\pi(1)}, \ldots, Y_{\pi(n)}\right),
$$

where $\left(C_{1}, \ldots, C_{n}\right) \in \operatorname{EMP}\left(\mu_{m}, n\right),\left(Y_{1}, \ldots, Y_{n}\right) \in\left(\mathbb{R}^{m-1}\right)^{n}$, and $\pi \in \mathfrak{S}_{n}$. These actions are introduced in such a way that the map $f_{\mathcal{M}}$ becomes an $\mathfrak{S}_{n}$-equivariant map. The image of the map $f_{\mathcal{M}}$ is a subset of the affine set $V \subseteq \mathbb{R}^{(m-1) \times n} \cong$ $\left(\mathbb{R}^{m-1}\right)^{n}$ given by

$$
V=\left\{\left(y_{j k}\right) \in \mathbb{R}^{(m-1) \times n}: \sum_{k=1}^{n} y_{j k}=\mu_{j}\left(\mathbb{R}^{d}\right) \text { for all } 1 \leqslant j \leqslant m-1\right\} \cong \mathbb{R}^{(m-1) \times(n-1)} .
$$

Consequently, we can assume that $f_{\mathcal{M}}: \operatorname{EMP}\left(\mu_{m}, n\right) \longrightarrow V \subseteq \mathbb{R}^{(m-1) \times n}$. 
Let $1 \leqslant i \leqslant n$, and let $I \subseteq[m-1]$ be a subset of cardinality $|I|=m-c+1$, where $[m-1]$ denotes the set of integers $\{1,2, \ldots, m-1\}$. Consider the subspace $L_{i, I}$ of $V$ given by

$$
L_{i, I}:=\left\{\left(y_{j k}\right) \in V: y_{r i}=0 \text { for every } r \in I\right\},
$$

and the associated arrangement

$$
\mathcal{A}=\mathcal{A}(m, n, c):=\left\{L_{i, I}: 1 \leqslant i \leqslant n, I \subseteq[m-1],|I|=m-c+1\right\} .
$$

The arrangement $\mathcal{A}$ is an $\mathfrak{S}_{n}$-invariant affine arrangement in $\mathbb{R}^{(m-1) \times n}$, meaning that $\pi \cdot L_{i, I} \in \mathcal{A}$ for every $\pi \in \mathfrak{S}_{n}$. Now we explain the key property of the arrangement $\mathcal{A}$. Let $\left(C_{1}, \ldots, C_{n}\right)$ be a convex partition of $\mathbb{R}^{d}$ that equiparts $\mu_{m}$ with a property that at least one of the subsets $C_{1}, \ldots, C_{n}$ has positive measure with respect to at most $c-1$ of the measures $\mu_{1}, \ldots, \mu_{m}$, which means that $\left(C_{1}, \ldots, C_{n}\right)$ is not a partition we are searching for. Since, by construction $\mu_{m}\left(C_{i}\right)>0$ for every $1 \leqslant i \leqslant n$, it follows that at least one of the subsets $C_{1}, \ldots, C_{n}$ has positive measure with respect to at most $c-2$ of the measures $\mu_{1}, \ldots, \mu_{m-1}$. Then there is a column of the matrix

$$
f_{\mathcal{M}}\left(C_{1}, \ldots, C_{n}\right) \in V \subseteq \mathbb{R}^{(m-1) \times n}
$$

with at most $c-2$ positive coordinates. In other words, there is a column of the matrix $f_{\mathcal{M}}\left(C_{1}, \ldots, C_{n}\right)$ with at least $m-c+1$ zeros, and consequently the matrix $f_{\mathcal{M}}\left(C_{1}, \ldots, C_{n}\right)$ is an element of the union $\bigcup \mathcal{A}:=\bigcup_{L_{i, I} \in \mathcal{A}} L_{i, I}$ of the arrangement $\mathcal{A}$.

Let us now assume that for integers $d \geqslant 2, m \geqslant 2, n \geqslant 1$, and $c \geqslant 1$, there exists a collection $\mathcal{M}=\left(\mu_{1}, \ldots, \mu_{m}\right)$ of absolutely continuous positive finite measures in $\mathbb{R}^{d}$ such that in every convex partition $\left(C_{1}, \ldots, C_{n}\right)$ of $\mathbb{R}^{d}$ that equiparts $\mu_{m}$ there is at least one subset $C_{k}$ that does not have positive measure with respect to at least $c$ measures, or equivalently it has measure zero with respect to at least $m-c+1$ of the measures $\mu_{1}, \ldots, \mu_{m}$. Consequently, $f_{\mathcal{M}}\left(C_{1}, \ldots, C_{n}\right) \in \bigcup \mathcal{A}$ for every convex partition $\left(C_{1}, \ldots, C_{n}\right)$ of $\mathbb{R}^{d}$ that equiparts the measure $\mu_{m}$. In particular, this means that the $\mathfrak{S}_{n}$-equivariant map $f_{\mathcal{M}}$ factors as follows

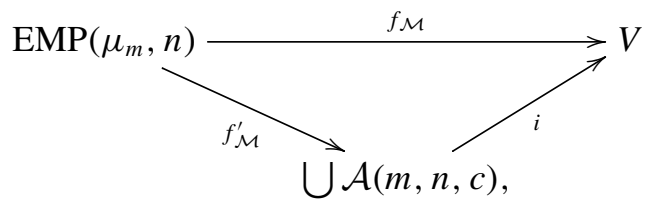

where $i: \cup \mathcal{A} \longrightarrow V$ is the inclusion and $f_{\mathcal{M}}^{\prime}: \operatorname{EMP}\left(\mu_{m}, n\right) \longrightarrow \bigcup \mathcal{A}$ is the $\mathfrak{S}_{n}$-equivariant map obtained from $f_{\mathcal{M}}$ by restricting the codomain. Thus, we have proved the following theorem. 
THEOREM 10. Let $d \geqslant 2, m \geqslant 2, n \geqslant 1$, and $c \geqslant 2$ be integers, and let $\mathcal{M}=$ $\left(\mu_{1}, \ldots, \mu_{m}\right)$ be a collection of absolutely continuous positive finite measures on $\mathbb{R}^{d}$. If there is no $\mathfrak{S}_{n}$-equivariant map

$$
\operatorname{EMP}\left(\mu_{m}, n\right) \longrightarrow \bigcup \mathcal{A}(m, n, c),
$$

then there exists a convex partition $\left(C_{1}, \ldots, C_{n}\right)$ of $\mathbb{R}^{d}$ that equiparts the measure $\mu_{m}$ with the additional property that each of the subsets $C_{i}$ has positive measure with respect to at least $c$ of the measures $\mu_{1}, \ldots, \mu_{m}$, that is

$$
\mu_{m}\left(C_{1}\right)=\cdots=\mu_{m}\left(C_{n}\right)=\frac{1}{n} \mu_{m}\left(\mathbb{R}^{d}\right)
$$

and

$$
\#\left\{j: 1 \leqslant j \leqslant m, \mu_{j}\left(C_{i}\right)>0\right\} \geqslant c
$$

for every $1 \leqslant i \leqslant n$.

\subsection{Existence of an equipartition of the sum of measures from nonexistence} of a map. As we have already mentioned, the CS/TM scheme needed for proving Theorem 4 is very similar to the one presented in Section 2.1. Nevertheless, it will be developed separately here.

Let $d \geqslant 2, m \geqslant 2, n \geqslant 1$, and $c \geqslant 2$ be integers, and let $\mathcal{M}=\left(\mu_{1}, \ldots, \mu_{m}\right)$ be a collection of absolutely continuous positive finite measures on $\mathbb{R}^{d}$. Denote by $\mu$ the sum of the measures $\mu_{1}, \ldots, \mu_{m}$, that is $\mu:=\sum_{j=1}^{m} \mu_{j}$.

Similarly as in Section 2.1, we define a continuous map $\tilde{f}_{\mathcal{M}}: \operatorname{EMP}(\mu, n) \longrightarrow$ $\mathbb{R}^{m \times n}$ as

$$
\left(C_{1}, \ldots, C_{n}\right) \longmapsto\left(\begin{array}{cccc}
\mu_{1}\left(C_{1}\right) & \mu_{1}\left(C_{2}\right) & \ldots & \mu_{1}\left(C_{n}\right) \\
\mu_{2}\left(C_{1}\right) & \mu_{2}\left(C_{2}\right) & \ldots & \mu_{2}\left(C_{n}\right) \\
\vdots & \vdots & \ddots & \vdots \\
\mu_{m}\left(C_{1}\right) & \mu_{m}\left(C_{2}\right) & \ldots & \mu_{m}\left(C_{n}\right)
\end{array}\right),
$$

where the domain of the map $\widetilde{f}_{\mathcal{M}}$ is the space of all convex partitions of $\mathbb{R}^{d}$ that equipart the measure $\mu$. The $\operatorname{map}_{\widetilde{f}} \widetilde{f}_{\mathcal{M}}$ is $\mathfrak{S}_{n}$-equivariant by construction. Furthermore, the image of the map $\widetilde{f}_{\mathcal{M}}$ is a subset of the affine set $\widetilde{V} \subseteq \mathbb{R}^{m \times n}$ given by

$$
\begin{array}{r}
\widetilde{V}=\left\{\left(y_{j k}\right) \in \mathbb{R}^{m \times n}: \sum_{k=1}^{n} y_{j k}=\mu_{j}\left(\mathbb{R}^{d}\right) \text { for every } 1 \leqslant j \leqslant m,\right. \\
\left.\sum_{j=1}^{m} y_{j k}=\frac{1}{n} \mu\left(\mathbb{R}^{d}\right) \text { for every } 1 \leqslant k \leqslant n\right\} .
\end{array}
$$


Now we define an affine arrangement that resembles the arrangement $\mathcal{A}$ from Section 2.1. Let $1 \leqslant i \leqslant n$, and let $I \subseteq[m]$ be a subset of cardinality $|I|=$ $m-c+1$. Consider the subspace $\widetilde{L}_{i, I}$ of $\widetilde{V}$ given by

$$
\widetilde{L}_{i, I}:=\left\{\left(y_{j k}\right) \in \widetilde{V}: y_{r, i}=0 \text { for every } r \in I\right\},
$$

and the associated $\mathfrak{S}_{n}$-invariant arrangement

$$
\widetilde{\mathcal{A}}=\widetilde{\mathcal{A}}(m, n, c):=\left\{\widetilde{L}_{i, I}: 1 \leqslant i \leqslant n, I \subseteq[m],|I|=m-c+1\right\} .
$$

Following the steps from Section 2.1, we study the key property of the arrangement $\widetilde{\mathcal{A}}$. Let $\left(C_{1}, \ldots, C_{n}\right)$ be a convex partition of $\mathbb{R}^{d}$ that does not satisfy the property asked in Theorem 4. More precisely, assume that for some $i$ the subset $C_{i}$ has positive measure with respect to at most $c-1$ of the measures $\mu_{1}$, $\ldots, \mu_{m}$. This means that the $i$ th column of the matrix $\widetilde{f}_{\mathcal{M}}\left(C_{1}, \ldots, C_{n}\right) \in \mathbb{R}^{m \times n}$ has at least $m-c+1$ zeros. In other words, $\widetilde{f}_{\mathcal{M}}\left(C_{1}, \ldots, C_{n}\right) \in \cup \widetilde{\mathcal{A}}$. Therefore, we have obtained the following theorem.

THEOREM 11. Let $d \geqslant 2, m \geqslant 2, n \geqslant 1$, and $c \geqslant 2$ be integers, and let $\mathcal{M}=$ $\left(\mu_{1}, \ldots, \mu_{m}\right)$ be a collection of absolutely continuous positive finite measures on $\mathbb{R}^{d}$. If there is no $\mathfrak{S}_{n}$-equivariant map

$$
\operatorname{EMP}(\mu, n) \longrightarrow \bigcup \tilde{\mathcal{A}}(m, n, c)
$$

then there exists a convex partition $\left(C_{1}, \ldots, C_{n}\right)$ of $\mathbb{R}^{d}$ that equiparts the measure $\mu=\mu_{1}+\cdots+\mu_{m}$ with the additional property that each of the subsets $C_{i}$ has positive measure with respect to at least $c$ of the measures $\mu_{1}, \ldots, \mu_{m}$, that is

$$
\mu\left(C_{1}\right)=\cdots=\mu\left(C_{n}\right)=\frac{1}{n} \mu\left(\mathbb{R}^{d}\right),
$$

and

$$
\#\left\{j: 1 \leqslant j \leqslant m, \mu_{j}\left(C_{i}\right)>0\right\} \geqslant c
$$

for every $1 \leqslant i \leqslant n$.

\section{Nonexistence of the equivariant maps}

This section is devoted to the proof of (non)-existence of equivariant maps from the space of regular convex partitions to appropriate affine arrangements. In Section 3.1, we consider the existence of an $\mathfrak{S}_{n}$-equivariant map $\operatorname{EMP}\left(\mu_{m}, n\right)$ $\longrightarrow \cup \mathcal{A}(m, n, c)$, whereas in Section 3.2, we focus on the existence of an $\mathfrak{S}_{n}$-equivariant map $\operatorname{EMP}(\mu, n) \longrightarrow \bigcup \widetilde{\mathcal{A}}(m, n, c)$ for different values of integer parameters $d, m, n$ and $c$. 
3.1. Nonexistence of an $\mathfrak{S}_{n}$-equivariant map $\operatorname{EMP}\left(\mu_{m}, n\right) \longrightarrow \bigcup \mathcal{A}(m, n, c)$. In order to prove the (non-)existence of an $\mathfrak{S}_{n}$-equivariant map

$$
\operatorname{EMP}\left(\mu_{m}, n\right) \longrightarrow \bigcup \mathcal{A}(m, n, c)
$$

we first construct various equivariant maps and prove a few auxiliary lemmas. In the following we use particular tools from the theory of homotopy colimits; for further details on these methods, consult for example [7], [19], or [17].

Let $X$ be a topological space and let $n \geqslant 1$ be an integer. The ordered configuration space $\operatorname{Conf}(X, n)$ of $n$ ordered pairwise distinct points of $X$ is the space

$$
\operatorname{Conf}(X, n):=\left\{\left(x_{1}, \ldots, x_{n}\right) \in X^{n} \mid x_{i} \neq x_{j} \text { for all } 1 \leqslant i<j \leqslant n\right\} .
$$

It was shown in [6, Section 2] that a subspace of $\operatorname{EMP}\left(\mu_{m}, n\right)$ consisting only of regular convex partitions can be parametrized by the configuration space $\operatorname{Conf}\left(\mathbb{R}^{d}, n\right)$. In particular, we have the following lemma.

LEMMA 12. There exists an $\mathfrak{S}_{n}$-equivariant map

$$
\alpha: \operatorname{Conf}\left(\mathbb{R}^{d}, n\right) \longrightarrow \operatorname{EMP}\left(\mu_{m}, n\right) .
$$

Let $P:=P(\mathcal{A})$ denote the intersection poset of the arrangement $\mathcal{A}=$ $\mathcal{A}(m, n, c)$, ordered by the reverse inclusion. The elements of the poset $P$ are nonempty intersections of subspaces in $\mathcal{A}$, thus they are of the form

$p_{\Lambda}:=\bigcap_{(i, I) \in \Lambda} L_{i, I}=\left\{\left(y_{j k}\right) \in V \subseteq \mathbb{R}^{(m-1) \times n}: y_{j i}=0\right.$, for all $1 \leqslant i \leqslant n$ and $\left.j \in I_{i}\right\}$,

where $\Lambda \subseteq[n] \times\left(\begin{array}{c}{[m-1]} \\ m-c+1\end{array}\right)$ and $I_{i}:=\bigcup_{(i, I) \in \Lambda} I$. Observe that sets $I_{i}$ can be empty. Alternatively, each poset element $p_{\Lambda}$ can be presented as an $(m-1) \times n$ matrix $\left(a_{j k}\right)$, where $a_{j k}=0$ if and only if $j \in I_{k}$. In other words, a coordinate $a_{j k}$ in the matrix presentation of $p_{\Lambda}$ equals zero if and only if $y_{j k}=0$ for every element $\left(y_{j k}\right) \in p_{\Lambda}$. An example of the poset $P(\mathcal{A})$ for parameters $n=2, m=4$ and $c=3$ is shown in Figure 1.

Let $\mathcal{C}$ be the $P$-diagram that corresponds to the arrangement $\mathcal{A}=\mathcal{A}(m, n, c)$, that is $\mathcal{C}\left(p_{\Lambda}\right):=p_{\Lambda}$ and $\mathcal{C}\left(p_{\Lambda^{\prime}} \supseteq p_{\Lambda^{\prime \prime}}\right): p_{\Lambda^{\prime \prime}} \longrightarrow p_{\Lambda^{\prime}}$ is the inclusion, see [19, Section 2.1]. The Equivariant Projection Lemma [17, Lemma 2.1] implies the following.

\section{LEMMA 13. The projection map}

$$
\operatorname{hocolim}_{P(\mathcal{A})} \mathcal{C} \longrightarrow \operatorname{colim}_{P(\mathcal{A})} \mathcal{C}=\bigcup \mathcal{A}
$$




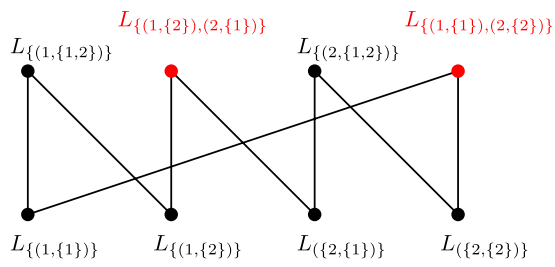

Figure 1 . Hasse diagram of the poset $P(\mathcal{A}(4,2,3))$.

is an $\mathfrak{S}_{n}$-equivariant homotopy equivalence. In particular, there exists an $\mathfrak{S}_{n}$ equivariant map

$$
\beta: \bigcup \mathcal{A} \longrightarrow \operatorname{hocolim}_{P(\mathcal{A})} \mathcal{C}
$$

Now, let $Q$ be the face poset of the $(n-1)$-dimensional simplex where the order is given by the inclusion. Define the monotone map $\varphi: P \longrightarrow Q$ by

$$
\varphi\left(p_{\Lambda}\right):=\{i \in[n]:(i, I) \in \Lambda \text { for some } I \subseteq[m-1]\} .
$$

Thus, $\varphi$ maps an element $p_{\Lambda}$ to the set of indices of its columns that contain zeros. It is important to notice that $\varphi$ does not have to be surjective, and therefore we set $Q^{\prime}:=\varphi(P) \subseteq Q$.

Next we consider the homotopy pushdown $\mathcal{D}$ of the diagram $\mathcal{C}$ along the map $\varphi$ over $Q^{\prime}$, see [19, Section 3.2]. This means that for $q \in Q^{\prime}$

$$
\mathcal{D}(q):=\left.\operatorname{hocolim}_{\varphi^{-1}\left(Q_{\geqslant q}^{\prime}\right)} \mathcal{C}\right|_{\varphi^{-1}\left(Q_{\geqslant q}^{\prime}\right)} \simeq \Delta\left(\varphi^{-1}\left(Q_{\geqslant q}^{\prime}\right)\right),
$$

and for every $q \geqslant r$ in $Q^{\prime}$ the map $\mathcal{D}(q \geqslant r): \mathcal{D}(q) \longrightarrow \mathcal{D}(r)$ is the corresponding inclusion. The next result follows from the Homotopy Pushdown Lemma [19, Proposition 3.12] adapted to the equivariant setting.

LEMMA 14. There is an $\mathfrak{S}_{n}$-equivariant homotopy equivalence

$$
\operatorname{hocolim}_{Q^{\prime}} \mathcal{D} \longrightarrow \operatorname{hocolim}_{P(\mathcal{A})} \mathcal{C} .
$$

In particular, there exists an $\mathfrak{S}_{n}$-equivariant map

$$
\gamma: \operatorname{hocolim}_{P(\mathcal{A})} \mathcal{C} \longrightarrow \operatorname{hocolim}_{Q^{\prime}} \mathcal{D} \text {. }
$$

We introduce another $Q^{\prime}$-diagram $\mathcal{E}$ by setting for $q \in Q^{\prime}$ that

$$
\mathcal{E}(q):= \begin{cases}\mathcal{D}(\hat{1}) \simeq \Delta\left(\varphi^{-1}(\{\hat{1}\})\right), & \text { if } q=\hat{1} \in Q^{\prime} \text { is the maximum of } Q, \\ \text { pt }, & \text { otherwise },\end{cases}
$$


and for every $q \geqslant r$ in $Q^{\prime}$ the map $\mathcal{E}(q \geqslant r)$ to be the constant map. In addition, we define a morphism of diagrams $(\Psi, \psi): \mathcal{D} \longrightarrow \mathcal{E}$, where $\psi: Q^{\prime} \longrightarrow Q^{\prime}$ is the identity map, and $\Psi(q): \mathcal{D}(q) \longrightarrow \mathcal{E}(q)$ is the identity map when $q$ is the maximal element, and constant map otherwise. The morphism $(\Psi, \psi)$ of diagrams induces an $\mathfrak{S}_{n}$-equivariant map between associated homotopy colimits, consult [19, Section 3]. Thus, we have established the following.

LEMMA 15. There exists an $\mathfrak{S}_{n}$-equivariant map

$$
\delta: \operatorname{hocolim}_{Q^{\prime}} \mathcal{D} \longrightarrow \operatorname{hocolim}_{Q^{\prime}} \mathcal{E} .
$$

In the final lemma we describe the hocolim $Q_{Q^{\prime}} \mathcal{E}$ up to an $\mathfrak{S}_{n}$-equivariant homotopy. First note that if $q, r \in Q$ are such that $q \geqslant r$ and $q \in Q^{\prime}$, then $r \in Q^{\prime}$. In particular, if $\hat{1} \in Q^{\prime}$, then $Q^{\prime}=Q$, where $\hat{1}$ is the maximum of $Q$.

LEMMA 16.

(i) If $\hat{1} \in Q^{\prime}$, that is $Q^{\prime}=Q$, then there exists an $\mathfrak{S}_{n}$-equivariant homotopy equivalence

$$
\operatorname{hocolim}_{Q^{\prime}} \mathcal{E} \simeq \Delta\left(Q^{\prime} \backslash\{\hat{1}\}\right) * \Delta\left(\varphi^{-1}(\{\hat{1}\})\right)
$$

where $\hat{1}$ is the maximum of $Q$, and $\operatorname{dim}\left(\Delta\left(\varphi^{-1}(\{\hat{1}\})\right)\right)=n c-m-2 n+1$. In particular, there exists an $\mathfrak{S}_{n}$-equivariant map

$$
\eta: \operatorname{hocolim}_{Q^{\prime}} \mathcal{E} \longrightarrow \Delta\left(Q^{\prime} \backslash\{\hat{1}\}\right) * \Delta\left(\varphi^{-1}(\{\hat{1}\})\right) .
$$

(ii) If $\hat{1} \notin Q^{\prime}$ then there exists an $\mathfrak{S}_{n}$-equivariant homotopy equivalence

$$
\operatorname{hocolim}_{Q^{\prime}} \mathcal{E} \simeq \Delta\left(Q^{\prime}\right),
$$

where $\operatorname{dim}\left(\Delta\left(Q^{\prime}\right)\right) \leqslant n-2$. In particular, there exists an $\mathfrak{S}_{n}$-equivariant map

$$
\eta: \operatorname{hocolim}_{Q^{\prime}} \mathcal{E} \longrightarrow \Delta\left(Q^{\prime}\right)
$$

Proof. (i) Let us first consider the case when $\hat{1} \in Q^{\prime}$. Then, since all the maps of the diagram $\mathcal{E}$ are constant maps, the Wedge lemma [19, Lemma 4.9] yields a homotopy equivalence

$$
\operatorname{hocolim}_{Q^{\prime}} \mathcal{E} \simeq \bigvee_{q \in Q^{\prime}}\left(\Delta\left(Q_{<q}^{\prime}\right) * \mathcal{E}(q)\right) \vee \Delta\left(Q^{\prime}\right) \simeq \Delta\left(Q^{\prime} \backslash\{\hat{1}\}\right) * \Delta\left(\varphi^{-1}(\{\hat{1}\})\right) .
$$

Here we use that $\Delta\left(Q^{\prime}\right) \simeq$ pt because $Q^{\prime}$ has the maximum. Furthermore, since for $q \neq \hat{1}$ all the spaces $\mathcal{E}(q)$ are points, this homotopy equivalence is an $\mathfrak{S}_{n}$-equivariant homotopy equivalence. 
The poset $\varphi^{-1}(\{\hat{1}\})$ consists of all points $p_{\Lambda} \in P$ that correspond to matrices which have zeros in all columns. Since it is a subposet of $P(\mathcal{A})$, every element of $\varphi^{-1}(\{\hat{1}\})$ must contain at least $m-c+1$ zeros in each column and at most $n-1$ zeros in each row. The partial order is given by

$$
p_{\Lambda} \leqslant p_{\Lambda^{\prime}} \Longleftrightarrow(\forall j \in[m-1])(\forall k \in[n]) a_{j k}=0 \Rightarrow a_{j k}^{\prime}=0,
$$

where $p_{\Lambda}=\left(a_{j k}\right)$ and $p_{\Lambda^{\prime}}=\left(a_{j k}^{\prime}\right)$. Maximal chains in the poset $\varphi^{-1}(\hat{1})$ can be obtained by removing zeros from a maximal element $p_{\Lambda}$ one by one, taking care that there must be at least $m-c+1$ zeros in each column. Maximal elements of $\varphi^{-1}(\hat{1})$ have exactly one nonzero element in each row, thus $(m-1)(n-1)$ zeros. Since $\hat{1} \in Q^{\prime}$ the minimal elements of the poset $\varphi^{-1}(\hat{1})$ have $m-c+1$ zeros in each column, thus $n(m-c+1)$ zeros. Therefore, the length of a maximal chain in $\varphi^{-1}(\hat{1})$, and consequently the dimension of its order complex $\Delta\left(\varphi^{-1}(\hat{1})\right)$, is $n c-m-2 n+1$. In particular, we obtained that when $\hat{1} \in Q^{\prime}$ then $n c-m-$ $2 n+1 \geqslant 0$, or equivalently $n(c-2)+1 \geqslant m$.

(ii) Let $\hat{1} \notin Q^{\prime}$. Then using the inclusion-exclusion principle it is not hard to see that $n(c-2)+1<m$. Again, the Wedge Lemma [19, Lemma 4.9] yields a homotopy equivalence

$$
\operatorname{hocolim}_{Q^{\prime}} \mathcal{E} \simeq \bigvee_{q \in Q^{\prime}}\left(\Delta\left(Q_{<q}^{\prime}\right) * \mathcal{E}(q)\right) \vee \Delta\left(Q^{\prime}\right) \simeq \Delta\left(Q^{\prime}\right),
$$

since now all the spaces $\mathcal{E}(q)$ are points for $q \in Q^{\prime}$.

From the assumption $\hat{1} \notin Q^{\prime}$ we get that $Q^{\prime} \subseteq Q \backslash\{\hat{1}\}$ and consequently $\Delta\left(Q^{\prime}\right) \subseteq \Delta(Q \backslash\{\hat{1}\})$. On the other hand $\Delta(Q \backslash\{\hat{1}\})$ is homeomorphic with the boundary of an $(n-1)$-dimensional simplex and so $\operatorname{dim}\left(\Delta\left(Q^{\prime}\right)\right) \leqslant n-2$.

In the example for parameters $n=2, m=4, c=3$, the poset $\varphi^{-1}(\{\hat{1}\})$ consists of two points $L_{\{(1,\{2\}),(2,\{1\})\}}$ and $L\{(1,\{1\}),(2,\{2\})\}$ with no relations between them, as shown in red in Figure 1.

THEOREM 17. Let $d \geqslant 2, m \geqslant 2$, and $c \geqslant 2$ be integers, and let $n=p^{k} \geqslant 2$ be a prime power. If

$$
m \geqslant n(c-d)+\frac{d n}{p}-\frac{n}{p}+1,
$$

then there is no continuous $\mathfrak{S}_{n}$-equivariant map

$$
\operatorname{EMP}\left(\mu_{m}, n\right) \longrightarrow \bigcup \mathcal{A}(m, n, c),
$$

where $\mu_{m}$ is a finite absolutely continuous measure on $\mathbb{R}^{d}$, and the affine arrangement $\mathcal{A}(m, n, c)$ is as defined in (1). 
Proof. Let $n=p^{k}$ be a prime power. Denote by $G \cong(\mathbb{Z} / p)^{k}$ a subgroup of the symmetric group $\mathfrak{S}_{n}$ given by the regular embedding (reg) : $G \longrightarrow \mathfrak{S}_{n}$, for more details see for example [1, Example III.2.7].

In order to prove the nonexistence of an $\mathfrak{S}_{n}$-equivariant map (3), we proceed by contradiction. Let $f: \operatorname{EMP}\left(\mu_{m}, n\right) \longrightarrow \bigcup \mathcal{A}(m, n, c)$ be a continuous $\mathfrak{S}_{n}$-equivariant map. Then from Lemmas $12,13,14,15$ and 16 we get the following composition of $\mathfrak{S}_{n}$-equivariant maps

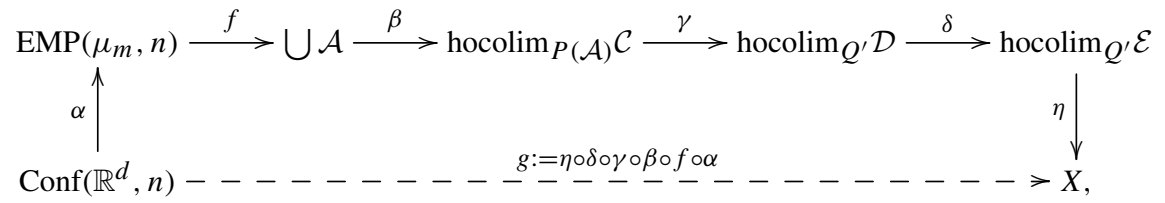

where

$$
X:= \begin{cases}\Delta\left(Q^{\prime} \backslash\{\hat{1}\}\right) * \Delta\left(\varphi^{-1}(\{\hat{1}\})\right), & \text { if } \hat{1} \in Q^{\prime}, \\ \Delta\left(Q^{\prime}\right), & \text { if } \hat{1} \notin Q^{\prime}\end{cases}
$$

Thus, the existence of an $\mathfrak{S}_{n}$-equivariant map $f: \operatorname{EMP}\left(\mu_{m}, n\right) \longrightarrow \bigcup \mathcal{A}$ implies the existence of an $\mathfrak{S}_{n}$-equivariant map $g: \operatorname{Conf}\left(\mathbb{R}^{d}, n\right) \longrightarrow C$. We will reach contradiction with the assumption that the map $f$ exists by proving that the map $g$ cannot exist. More precisely, we will show that there cannot exist a $G$-equivariant map

$$
\operatorname{Conf}\left(\mathbb{R}^{d}, n\right) \longrightarrow X .
$$

First, we consider a degenerate case when $X$ is not path-connected. This can happen only when $n=2$ and $\hat{1} \notin Q^{\prime}$. Then $X=\Delta\left(Q^{\prime}\right) \cong S^{0}$ where the action of the group $\mathfrak{S}_{2}=G \cong \mathbb{Z} / 2$ on $S^{0}$ is free. Now, if a $G$-equivariant map (4) exists then it is a continuous surjection of path-connected space $\operatorname{Conf}\left(\mathbb{R}^{d}, 2\right), d \geqslant 2$, onto the sphere $S^{0}$; a contradiction. This concludes the proof of the theorem in this case.

We proceed by assume that $X$ is path-connected. That is, $n \geqslant 3$, or $n=2$ and $\hat{1} \in Q^{\prime}$. We start with a continuous $\mathfrak{S}_{n}$ and also $G$-equivariant map $g: \operatorname{Conf}\left(\mathbb{R}^{d}, n\right)$ $\longrightarrow X$. The map $g$ induces a morphism between Borel construction fibrations:

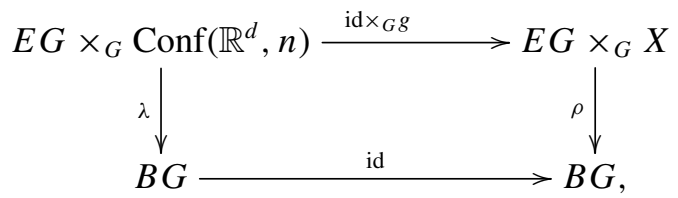

which in turn induces a morphism between corresponding Serre spectral sequences $E_{*}^{* * *}(g): E_{*}^{*, *}(\rho) \longrightarrow E_{*}^{* * *}(\lambda)$. The crucial property of the morphism $E_{*}^{* * *}(g)$ we use is that $E_{2}^{*, 0}(g)=$ id. A contradiction with the assumption that 
there is a map $g$, which is going to be obtained from an analysis of the morphism $E_{*}^{* * *}(g)$. For that we first describe the spectral sequences $E_{*}^{*, *}(\lambda)$ and $E_{*}^{*, *}(\rho)$.

The Serre spectral sequence of the fibration

$$
\operatorname{Conf}\left(\mathbb{R}^{d}, n\right) \longrightarrow E G \times{ }_{G} \operatorname{Conf}\left(\mathbb{R}^{d}, n\right) \longrightarrow B G
$$

has the $E_{2}$-term given by

$$
E_{2}^{i, j}(\lambda)=H^{i}\left(B G ; \mathcal{H}^{j}\left(\operatorname{Conf}\left(\mathbb{R}^{d}, n\right) ; \mathbb{F}_{p}\right)\right) \cong H^{i}\left(G ; H^{j}\left(\operatorname{Conf}\left(\mathbb{R}^{d}, n\right) ; \mathbb{F}_{p}\right)\right) .
$$

Here $H^{i}\left(B G ; \mathcal{H}^{j}\left(Y ; \mathbb{F}_{p}\right)\right)$ denotes the cohomology of $B G$ with local coefficients in $H^{j}\left(Y ; \mathbb{F}_{p}\right)$ determined by the action of the fundamental group of the base space $\pi_{1}(B G) \cong G$. The second description uses the fact that cohomology of the classifying space $B G$ of the group $G$ is by definition the cohomology of the group $G$ with coefficients in the $G$-module $H^{j}\left(\operatorname{Conf}\left(\mathbb{R}^{d}, n\right) ; \mathbb{F}_{p}\right)$. For more details on the cohomology with local coefficients, consult for example [11, Section 3.H]. The spectral sequence $E_{*}^{*, *}(\lambda)$ was completely determined in the case $k=1$, that is, $n=p$ a prime, by Cohen [8, Theorem 8.2] and recently in [4, Theorem 6.1]. A partial description of $E_{*}^{* * *}(\lambda)$ in the case $k \geqslant 2$ was given in [4, Theorems 6.3 and 7.1]. In particular, for $k=1$

$$
E_{2}^{*, *}(\lambda) \cong E_{3}^{*, *}(\lambda) \cong \cdots \cong E_{(d-1)(n-1)+1}^{*, *}(\lambda),
$$

and

$$
E_{(d-1)(n-1)+2}^{*, *}(\lambda) \cong \cdots \cong E_{\infty}^{*, *}(\lambda)
$$

while for $k \geqslant 2$

$$
E_{2}^{*, *}(\lambda) \cong E_{3}^{*, *}(\lambda) \cong \cdots \cong E_{(d-1)(n-n / p)+1}^{*, *}(\lambda) .
$$

In the second step we consider the Serre spectral sequence of the fibration

$$
X \longrightarrow E G \times_{G} X \longrightarrow B G
$$

whose $E_{2}$-term is given by

$$
E_{2}^{i, j}(\rho)=H^{i}\left(B G ; \mathcal{H}^{j}\left(X ; \mathbb{F}_{p}\right)\right) \cong H^{i}\left(G ; H^{j}\left(X ; \mathbb{F}_{p}\right)\right) .
$$

See Figure 2 for an illustration. We conclude the proof by considering two separate cases.

(a) Let $\hat{1} \in Q^{\prime}$, or equivalently $n c-m-2 n+1 \geqslant 0$. Then the simplicial complex $X=\Delta\left(Q^{\prime} \backslash\{\hat{1}\}\right) * \Delta\left(\varphi^{-1}(\{\hat{1}\})\right)$ is at most $(n c-m-n)$-dimensional, implying that $E_{2}^{i, j}(\rho)=0$ for all $j \geqslant n c-m-n+1$. Consequently all differentials $r_{r}$ for $r \geqslant n c-m-n+2$ vanish and so

$$
E_{n c-m-n+2}^{i, j}(\rho) \cong E_{n c-m-n+3}^{i, j}(\rho) \cong \cdots \cong E_{\infty}^{i, j}(\rho) .
$$




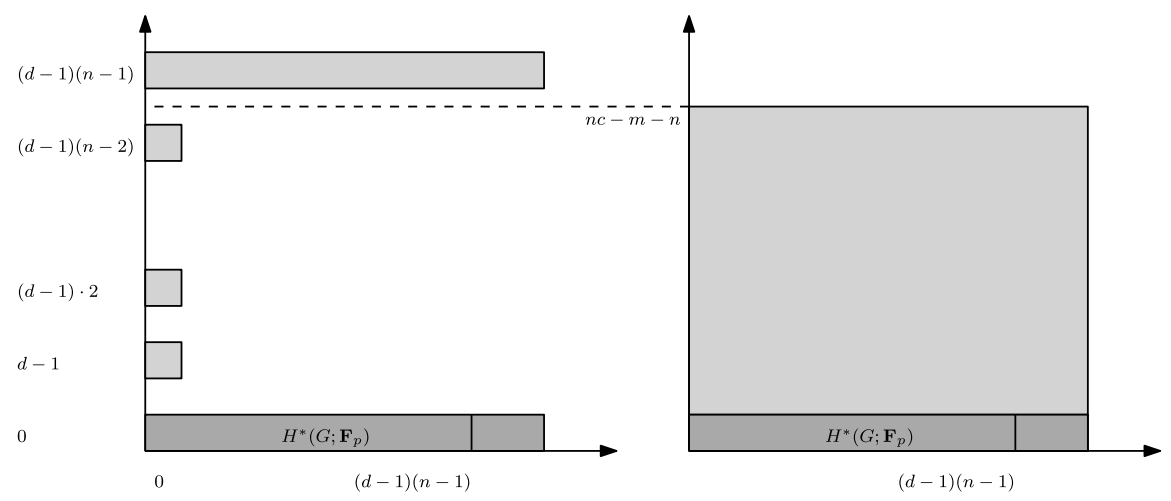

Figure 2. An illustration of $E_{2}^{*, *}(\lambda)$ and $E_{2}^{*, *}(\rho)$ in the case when $n=p$ is a prime.

Next, since the path-connected simplicial complex $X$ does not have fixed points with respect to the action of the elementary abelian group $G$, a consequence of the localization theorem [13, Corollary 1, p. 45] implies that $H^{*}\left(G ; \mathbb{F}_{p}\right) \cong E_{2}^{*, 0}(\rho) \nsucceq$ $E_{\infty}^{*, 0}(\rho)$. Having in mind (8) we conclude that

$$
H^{*}\left(G ; \mathbb{F}_{p}\right) \cong E_{2}^{*, 0}(\rho) \varsubsetneqq E_{n c-m-n+2}^{*, 0}(\rho) .
$$

For our proof, without loss of generality, we can assume that

$$
H^{*}\left(G ; \mathbb{F}_{p}\right) \cong E_{2}^{*, 0}(\rho) \cong E_{n c-m-n+1}^{*, 0}(\rho) \varsubsetneqq E_{n c-m-n+2}^{*, 0}(\rho) .
$$

Now, from the assumption on $m$, we deduce that for $k=1$

$$
(d-1)(n-1)+1 \geqslant n c-m-n+2,
$$

and for $k \geqslant 2$

$$
(d-1)\left(n-\frac{n}{p}\right)+1 \geqslant n c-m-n+2 .
$$

Hence the fact that $E_{2}^{*, 0}(g)=$ id, in combination with relations (5), (6), (7) and (9), yields a contradiction: the homomorphism $E_{n c-m-n+2}^{*, 0}(g)$ sends the zero to a nonzero element. This concludes the proof of the theorem in the case when $n c-2 n+1 \geqslant m$.

(b) Let $\hat{1} \notin Q^{\prime}$, or equivalently $n c-m-2 n+1<0$. The simplicial complex $X=\Delta\left(Q^{\prime}\right)$ is at most $(n-2)$-dimensional. Hence, $E_{2}^{i, j}(\rho)=0$ for all $j \geqslant n-1$, and

$$
E_{n}^{i, j}(\rho) \cong E_{n+1}^{i, j}(\rho) \cong \cdots \cong E_{\infty}^{i, j}(\rho)
$$


The simplicial complex $X$ is path-connected and without fixed points with respect to the action of the elementary abelian group $G$. Consequence of the localization theorem [13, Corollary 1, p. 45] implies that $H^{*}\left(G ; \mathbb{F}_{p}\right) \cong E_{2}^{*, 0}(\rho) \approx E_{\infty}^{*, 0}(\rho)$. From (10) we have that

$$
H^{*}\left(G ; \mathbb{F}_{p}\right) \cong E_{2}^{*, 0}(\rho) \varsubsetneqq E_{n}^{*, 0}(\rho) .
$$

For our proof, without loss of generality, we can assume that

$$
H^{*}\left(G ; \mathbb{F}_{p}\right) \cong E_{2}^{*, 0}(\rho) \cong E_{n-1}^{*, 0}(\rho) \varsubsetneqq E_{n}^{*, 0}(\rho) .
$$

Now, we need that for $k=1$

$$
(d-1)(n-1)+1 \geqslant n,
$$

and for $k \geqslant 2$

$$
(d-1)\left(n-\frac{n}{p}\right)+1 \geqslant n
$$

is satisfied. Indeed, these conditions are satisfied for $d \geqslant 2, p \geqslant 2$ and $n=p^{k}$. Thus, the fact that $E_{2}^{*, 0}(g)=$ id with (5), (7) and (11) gives a contradiction: the homomorphism $E_{n}^{*, 0}(g)$ sends the zero to a nonzero element. This concludes the proof of the theorem in the case when $n c-2 n+1<m$.

The previous proof can also be phrased in the language of the iterated index theory introduced by Volovikov in [18].

3.2. Nonexistence of an $\mathfrak{S}_{n}$-equivariant map $\operatorname{EMP}(\mu, n) \longrightarrow \bigcup \widetilde{\mathcal{A}}(m, n, c)$. Motivated by Theorem 11, in this section we prove the (non-)existence of a continuous $\mathfrak{S}_{n}$-equivariant map

$$
\operatorname{EMP}(\mu, n) \longrightarrow \bigcup \tilde{\mathcal{A}}(m, n, c)
$$

for different values of parameters $d, m, n$ and $c$. Following the structure of Section 3.1, we first prove a few auxiliary lemmas in order to arrive to the topological result, Theorem 11, at the end of this section.

Recalling that a subspace of $\operatorname{EMP}(\mu, n)$ consisting only of regular convex partitions can be identified with the configuration space $\operatorname{Conf}\left(\mathbb{R}^{d}, n\right)$, see [6, Section 2] for more details, we obtain the following lemma.

LEMMA 18. There exists an $\mathfrak{S}_{n}$-equivariant map

$$
\tilde{\alpha}: \operatorname{Conf}\left(\mathbb{R}^{d}, n\right) \longrightarrow \operatorname{EMP}(\mu, n) .
$$


Denote by $\widetilde{P}=P(\widetilde{\mathcal{A}})$ the intersection poset of the affine arrangement $\widetilde{\mathcal{A}}$. Its elements are given by $\widetilde{p}_{\Lambda}:=\bigcap_{(i, I) \in \Lambda} \widetilde{L}_{i, I}=\left\{\left(y_{j k}\right) \in \widetilde{V} \subseteq \mathbb{R}^{m \times n}: y_{j i}=0\right.$, for all $1 \leqslant i \leqslant n$ and $\left.j \in I_{i}\right\}$, where $\Lambda \subseteq[n] \times\left(\begin{array}{c}{[m]} \\ m-c+1\end{array}\right)$ and $I_{i}:=\bigcup_{(i, I) \in \Lambda} I$. An element $\widetilde{p}_{\Lambda}$ can also be seen as an $m \times n$ matrix $\left(a_{j k}\right)$, where $a_{j k}=0$ if and only if $j \in I_{k}$.

Next we consider a $\widetilde{P}$-diagram $\widetilde{\mathcal{C}}$ determined by the arrangement $\widetilde{\mathcal{A}}=$ $\widetilde{\mathcal{A}}(m, n, c)$. More precisely, we define $\widetilde{\mathcal{C}}\left(\widetilde{p}_{\Lambda}\right):=\widetilde{p}_{\Lambda}$ and $\widetilde{\mathcal{C}}\left(\widetilde{p}_{\Lambda^{\prime}} \supseteq \widetilde{p}_{\Lambda^{\prime \prime}}\right): \widetilde{p}_{\Lambda^{\prime \prime}} \longrightarrow$ $\tilde{p}_{\Lambda^{\prime}}$ to be the inclusion. The Equivariant Projection Lemma [17, Lemma 2.1] implies the following.

LEMMA 19. The projection map

$$
\operatorname{hocolim}_{\tilde{P}} \widetilde{\mathcal{C}} \longrightarrow \operatorname{colim}_{\tilde{P}} \widetilde{\mathcal{C}}=\bigcup \tilde{\mathcal{A}}
$$

is an $\mathfrak{S}_{n}$-equivariant homotopy equivalence. In particular, there exists an $\mathfrak{S}_{n}$ equivariant map

$$
\widetilde{\beta}: \bigcup \widetilde{\mathcal{A}} \longrightarrow \operatorname{hocolim}_{\tilde{P}} \widetilde{\mathcal{C}}
$$

Recall that $Q$ denotes the face poset of an $(n-1)$-dimensional simplex, and define a map $\widetilde{\varphi}: \widetilde{P} \rightarrow Q$ by

$$
\widetilde{\varphi}\left(\widetilde{p}_{\Lambda}\right):=\{i \in[n]:(i, I) \in \Lambda \text { for some } I \subseteq[m]\} .
$$

Additionally, denote the poset $\widetilde{\varphi}(\widetilde{P}) \subseteq Q$ by $Q^{\prime}$. Note that if $q, r \in Q$ are such that $q \in Q^{\prime}$ and $r \leqslant q$, then $r$ is also an element of $Q^{\prime}$. In particular, if $q=\hat{1}$ is the maximal element of $Q$ and $q \in Q^{\prime}$, then $Q^{\prime}=Q$.

Let $\widetilde{\mathcal{D}}$ be the homotopy pushdown of the diagram $\widetilde{\mathcal{C}}$ along the map $\widetilde{\varphi}$ over $Q^{\prime}$. This means that

$$
\widetilde{\mathcal{D}}(q):=\left.\operatorname{hocolim}_{\widetilde{\varphi}^{-1}\left(Q_{\geqslant q}^{\prime}\right)} \widetilde{\mathcal{C}}\right|_{\widetilde{\varphi}^{-1}\left(Q_{\geqslant q}^{\prime}\right)} \simeq \Delta\left(\widetilde{\varphi}^{-1}\left(Q_{\geqslant q}^{\prime}\right)\right)
$$

for $q \in Q^{\prime}$, and the map $\widetilde{\mathcal{D}}(q \geqslant r): \widetilde{\mathcal{D}}(q) \longrightarrow \widetilde{\mathcal{D}}(r)$ is the corresponding inclusion for every $q \geqslant r$ in $Q^{\prime}$. Once more, the Homotopy Pushdown Lemma [19, Proposition 3.12] adapted to equivariant setting yields the following fact.

LEMMA 20. There is an $\mathfrak{S}_{n}$-equivariant homotopy equivalence

$$
\operatorname{hocolim}_{Q^{\prime}} \widetilde{\mathcal{D}} \longrightarrow \operatorname{hocolim}_{\widetilde{P}} \widetilde{\mathcal{C}}
$$

In particular, there exists an $\mathfrak{S}_{n}$-equivariant map

$$
\tilde{\gamma}: \operatorname{hocolim}_{\widetilde{P}} \widetilde{\mathcal{C}} \longrightarrow \operatorname{hocolim}_{Q^{\prime}} \widetilde{\mathcal{D}}
$$


Finally, we consider another $Q^{\prime}$-diagram $\widetilde{\mathcal{E}}$ by setting for $q \in Q^{\prime}$ that

$$
\widetilde{\mathcal{E}}(q)= \begin{cases}\Delta\left(\widetilde{\varphi}^{-1}(\{\hat{1}\})\right), & \text { if } q=\hat{1} \in Q^{\prime} \text { is the maximum of } Q, \\ \mathrm{pt}, & \text { otherwise }\end{cases}
$$

and the map $\widetilde{\mathcal{E}}(q \geqslant r)$ to be the constant map for every $q \geqslant r$ in $Q^{\prime}$. Similarly as we have done it in Section 3.1, we define a morphism of diagrams $(\widetilde{\Psi}, \widetilde{\psi}): \widetilde{\mathcal{D}} \longrightarrow$ $\widetilde{\mathcal{E}}$, where $\widetilde{\psi}: Q^{\prime} \longrightarrow Q^{\prime}$ is the identity map, and $\widetilde{\Psi}(q): \widetilde{\mathcal{D}}(q) \longrightarrow \widetilde{\mathcal{E}}(q)$ is the identity map when $q=\hat{1}$ is the maximal element in $Q$, and constant map otherwise. Since the morphism $(\widetilde{\Psi}, \widetilde{\psi})$ of diagrams induces an $\mathfrak{S}_{n}$-equivariant map between associated homotopy colimits, we have established the following.

LEMMA 21. There exists an $\mathfrak{S}_{n}$-equivariant map

$$
\tilde{\delta}: \operatorname{hocolim}_{Q^{\prime}} \widetilde{\mathcal{D}} \longrightarrow \operatorname{hocolim}_{Q^{\prime}} \widetilde{\mathcal{E}}
$$

Just like in Section 3.1, the final lemma will describe the hocolim ${ }_{Q^{\prime}} \widetilde{\mathcal{E}}$ up to an $\mathfrak{S}_{n}$-equivariant homotopy.

\section{LEMMA 22.}

(i) If $\hat{1} \in Q^{\prime}$, that is if $Q^{\prime}=Q$, then there exists an $\mathfrak{S}_{n}$-equivariant homotopy equivalence

$$
\operatorname{hocolim}_{Q} \widetilde{\mathcal{E}} \simeq \Delta(Q \backslash\{\hat{1}\}) * \Delta\left(\widetilde{\varphi}^{-1}(\{\hat{1}\})\right)
$$

where $\hat{1}$ is the maximum of $Q$, and $\operatorname{dim}\left(\Delta\left(\widetilde{\varphi}^{-1}(\hat{1})\right)\right)=n c-n-\max \{m, n\}$. In particular, there exists an $\mathfrak{S}_{n}$-equivariant map

$$
\widetilde{\eta}: \operatorname{hocolim}_{Q} \widetilde{\mathcal{E}} \longrightarrow \Delta(Q \backslash\{\hat{1}\}) * \Delta\left(\widetilde{\varphi}^{-1}(\{\hat{1}\})\right) .
$$

(ii) If $\hat{1} \notin Q^{\prime}$ then there exists an $\mathfrak{S}_{n}$-equivariant homotopy equivalence

$$
\operatorname{hocolim}_{Q^{\prime}} \widetilde{\mathcal{E}} \simeq \Delta\left(Q^{\prime}\right),
$$

where $\operatorname{dim}\left(\Delta\left(Q^{\prime}\right)\right) \leqslant n-2$. In particular, there exists an $\mathfrak{S}_{n}$-equivariant map

$$
\widetilde{\eta}: \operatorname{hocolim}_{Q^{\prime}} \widetilde{\mathcal{E}} \longrightarrow \Delta\left(Q^{\prime}\right) \text {. }
$$

Proof. The proof of the claim (ii) is identical to the proof of the second part of Lemma 16. For the claim (i) it suffices to compute the dimension of the simplicial complex $\Delta\left(\widetilde{\varphi}^{-1}(\{\hat{1}\})\right)$, since the rest of the proof follows the lines of the proof of the first part of Lemma 16. 
The elements of the poset $\widetilde{\varphi}^{-1}(\{\hat{1}\})$ are presented by matrices $\widetilde{p}_{\Lambda}=\left(a_{j k}\right)$ that contain zeros in every column. The partial order is given by

$$
\tilde{p}_{\Lambda} \leqslant \tilde{p}_{\Lambda^{\prime}} \Longleftrightarrow(\forall j \in[m])(\forall k \in[n]) a_{j k}=0 \Rightarrow a_{j k}^{\prime}=0,
$$

where $\tilde{p}_{\Lambda}=\left(a_{j k}\right)$ and $\widetilde{p}_{\Lambda^{\prime}}=\left(a_{j k}^{\prime}\right)$ are elements of the poset $\widetilde{\varphi}^{-1}(\{\hat{1}\}) \subseteq \widetilde{P}$. Maximal chains in $\widetilde{\varphi}^{-1}(\{\hat{1}\})$ can be obtained by removing zeros one by one from a matrix that represents a maximal element, taking care of the fact that every column has to contain at least $m-c+1$ zeros. The maximal elements are presented by matrices that have at most $n-1$ zeros in each row, and at most $m-1$ zeros in each column. Thus, maximal elements are presented by matrices with $m n-\max (m, n)$ zeros. The minimal elements, on the other hand, are presented by matrices that contain $n(m-c+1)$ zeros. Therefore, the dimension of $\Delta\left(\widetilde{\varphi}^{-1}(\hat{1})\right)$ is $n c-n-\max \{m, n\} \geqslant 0$. Since $c \geqslant 2$, this implies that $n(c-1) \geqslant m$.

Now we are ready to prove the central result about the nonexistence of an $\mathfrak{S}_{n}$-equivariant map $\operatorname{EMP}(\mu, n) \longrightarrow \bigcup \widetilde{\mathcal{A}}$.

THEOREM 23. Let $d \geqslant 2, m \geqslant 2$, and $c \geqslant 2$ be integers, and let $n=p^{k} \geqslant 2$ be a prime power. If

(a) $n(c-1) \geqslant m$ and $\max \{m, n\} \geqslant n(c-d)+(d n / p)-(n / p)+n$, or

(b) $n(c-1)<m$,

then there is no $\mathfrak{S}_{n}$-equivariant map

$$
\operatorname{EMP}(\mu, n) \longrightarrow \bigcup \widetilde{\mathcal{A}}(m, n, c),
$$

where $\mu=\mu_{1}+\cdots+\mu_{m}$ is the sum of $m$ finite absolutely continuous measures on $\mathbb{R}^{d}$, and the affine arrangement $\widetilde{\mathcal{A}}(m, n, c)$ is as defined in $(2)$.

Proof. It is not surprising that this proof will follow the lines of the proof of Theorem 17. Let $n=p^{k}$ be a prime power and denote by $G \cong(\mathbb{Z} / p)^{k}$ a subgroup of the symmetric group $\mathfrak{S}_{n}$ given by the regular embedding (reg) $: G \longrightarrow \mathfrak{S}_{n}$.

The proof is by contradiction. Therefore, assume that $\widetilde{f}: \operatorname{EMP}(\mu, n) \longrightarrow$ $\cup \widetilde{\mathcal{A}}(m, n, c)$ is a continuous $\mathfrak{S}_{n}$-equivariant map. From Lemmas 18, 19, 20,21 and 22 we again get a composition of $\mathfrak{S}_{n}$-equivariant maps

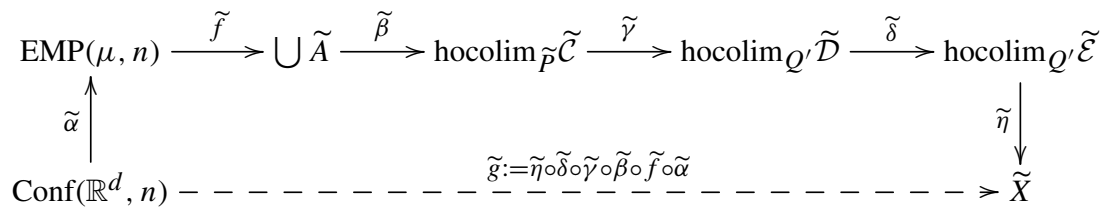


where

$$
\widetilde{X}:= \begin{cases}\Delta(Q \backslash\{\hat{1}\}) * \Delta\left(\widetilde{\varphi}^{-1}(\{\hat{1}\})\right), & \text { if } \hat{1} \in Q^{\prime}, \\ \Delta\left(Q^{\prime}\right), & \text { if } \hat{1} \notin Q^{\prime}\end{cases}
$$

It suffices to show that the map $\widetilde{g}$ cannot exist, since that would contradict the existence of the map $\widetilde{f}$. Actually, we will prove here that there is no continuous $G$-equivariant map

$$
\operatorname{Conf}\left(\mathbb{R}^{d}, n\right) \longrightarrow \widetilde{X}
$$

Again we first consider a degenerate case when $\widetilde{X}$ is not path-connected. That is, $n=2$ and $\hat{1} \notin Q^{\prime}$. Consequently $\widetilde{X}=\Delta\left(Q^{\prime}\right) \cong S^{0}$ where the action of the group $\mathfrak{S}_{2}=G \cong \mathbb{Z} / 2$ on $S^{0}$ is free. Now, if a $G$-equivariant map (13) exists then it is a continuous surjection of path-connected space $\operatorname{Conf}\left(\mathbb{R}^{d}, 2\right), d \geqslant 2$, onto the sphere $S^{0}$; a contradiction. This completes the proof of the theorem in this case.

Now assume that $\widetilde{X}$ is path-connected. That is, $n \geqslant 3$, or $n=2$ and $\hat{1} \in Q^{\prime}$. Consider a continuous map $\widetilde{g}: \operatorname{Conf}\left(\mathbb{R}^{d}, n\right) \longrightarrow \widetilde{X}$ which is $\mathfrak{S}_{n}$ and also $G$-equivariant. It induces a morphism between Borel construction fibrations:

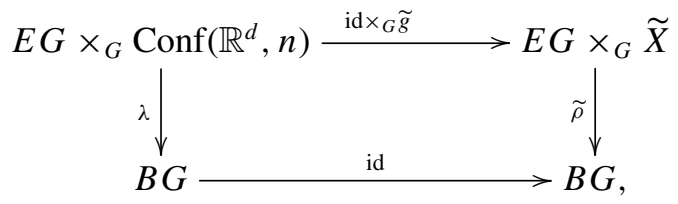

which furthermore induces a morphism between the corresponding Serre spectral sequences

$$
E_{*}^{*, *}(\widetilde{g}): E_{*}^{*, *}(\widetilde{\rho}) \longrightarrow E_{*}^{*, *}(\lambda) .
$$

Like in the proof of Theorem 17, we use the fact that $E_{2}^{*, 0}(\widetilde{g})=$ id. Next we analyse the morphism $E_{*}^{*, *}(\widetilde{g})$. Since the spectral sequence $E_{*}^{*, *}(\lambda)$ was already described in the proof of Theorem 17, we concentrate here on the spectral sequence $E_{*}^{*, *}(\widetilde{\rho})$.

The Serre spectral sequence of the fibration

$$
\widetilde{X} \longrightarrow E G \times_{G} \tilde{X} \longrightarrow B G
$$

has the $E_{2}$-term given by

$$
E_{2}^{i, j}(\widetilde{\rho})=H^{i}\left(B G ; \mathcal{H}^{j}\left(\widetilde{X} ; \mathbb{F}_{p}\right)\right) \cong H^{i}\left(G ; H^{j}\left(\widetilde{X} ; \mathbb{F}_{p}\right)\right)
$$

In order to conclude the proof we consider two separate cases.

(a) Let $\hat{1} \in Q^{\prime}$ and let $m$ satisfy the condition of the theorem. Then $n(c-1) \geqslant m$, so the simplicial complex

$$
\widetilde{X}=\Delta(Q \backslash\{\hat{1}\}) * \Delta\left(\widetilde{\varphi}^{-1}(\{\hat{1}\})\right)
$$


is at most $(n c-\max \{m, n\}-1)$-dimensional. This implies that $E_{2}^{i, j}(\widetilde{\rho})=0$ for all $j \geqslant n c-\max \{m, n\}$, and consequently,

$$
E_{n c-\max \{m, n\}+1}^{i, j}(\widetilde{\rho}) \cong E_{n c-\max \{m, n\}+2}^{i, j}(\widetilde{\rho}) \cong \cdots \cong E_{\infty}^{i, j}(\widetilde{\rho}) .
$$

Once more a consequence of the localization theorem [13, Corollary 1, p. 45] implies that $H^{*}\left(G ; \mathbb{F}_{p}\right) \cong E_{2}^{*, 0}(\widetilde{\rho}) ¥ E_{\infty}^{*, 0}(\widetilde{\rho})$, because the path-connected simplicial complex $\widetilde{X}$ does not have fixed points with respect to the action of the elementary abelian group $G$. Having in mind (14) we conclude that

$$
H^{*}\left(G ; \mathbb{F}_{p}\right) \cong E_{2}^{*, 0}(\widetilde{\rho}) ¥ E_{n c-\max \{m, n\}+1}^{*, 0}(\widetilde{\rho}) .
$$

For our proof, without loss of generality, we can assume that

$$
H^{*}\left(G ; \mathbb{F}_{p}\right) \cong E_{2}^{*, 0}(\widetilde{\rho}) \cong E_{n c-\max \{m, n\}-2}^{*, 0}(\widetilde{\rho}) \varsubsetneqq \mathcal{E}_{n c-\max \{m, n\}-1}^{*, 0}(\widetilde{\rho}) .
$$

Now, the assumption on $m$ and $n$, means for $k=1$

$$
(d-1)(n-1)+1 \geqslant n c-\max \{m, n\}+1,
$$

and for $k \geqslant 2$

$$
(d-1)\left(n-\frac{n}{p}\right)+1 \geqslant n c-\max \{m, n\}+1 .
$$

Therefore, the relations (5), (7) and (15), together with the fact that $E_{2}^{*, 0}(\widetilde{g})=$ id, yield a contradiction: the homomorphism $E_{n c-\max \{m, n\}+1}^{*, 0}(\widetilde{g})$ sends the zero to a nonzero element. This concludes the proof of the theorem in the case when $n(c-1) \geqslant m$.

(b) Let $\hat{1} \notin Q^{\prime}$, or equivalently $n(c-1)<m$. The simplicial complex $\tilde{X}=$ $\Delta\left(Q^{\prime}\right)$ is at most $(n-2)$-dimensional, by Lemma 22(ii). Thus, $E_{2}^{i, j}(\widetilde{\rho})=0$ for all $j \geqslant n-1$, and furthermore

$$
E_{n}^{i, j}(\widetilde{\rho}) \cong E_{n+1}^{i, j}(\widetilde{\rho}) \cong \cdots \cong E_{\infty}^{i, j}(\widetilde{\rho})
$$

For the same reason as above, we have $H^{*}\left(G ; \mathbb{F}_{p}\right) \cong E_{2}^{*, 0}(\widetilde{\rho}) \varsubsetneqq E_{\infty}^{*, 0}(\widetilde{\rho})$. This fact combined with the isomorphisms in (16) yields that

$$
H^{*}\left(G ; \mathbb{F}_{p}\right) \cong E_{2}^{*, 0}(\widetilde{\rho}) \varsubsetneqq E_{n}^{*, 0}(\widetilde{\rho}) .
$$

Again, without loss of generality, we can assume that

$$
H^{*}\left(G ; \mathbb{F}_{p}\right) \cong E_{2}^{*, 0}(\widetilde{\rho}) \cong E_{n-1}^{*, 0}(\widetilde{\rho}) \varsubsetneqq E_{n}^{*, 0}(\widetilde{\rho}) .
$$


In order to complete the proof we need that for $k=1$

$$
(d-1)(n-1)+1 \geqslant n,
$$

and for $k \geqslant 2$

$$
(d-1)\left(n-\frac{n}{p}\right)+1 \geqslant n .
$$

Indeed, both of these inequalities are satisfied, thus the fact that $E_{2}^{*, 0}(\widetilde{g})=$ id with (5), (7) and (17) gives a contradiction: the homomorphism $E_{n}^{*, 0}(\widetilde{g})$ sends the zero to a nonzero element. This concludes the proof of the theorem in the case when $n(c-1)<m$.

\section{Proofs}

Finally, in this section proofs of Theorems 2, 3, 4, 5, 7, 8, and 9 will be presented. The proofs of Theorems 2 and 7 are completely geometric and they do not involve any topological methods. The proofs of Theorems 5, 8, and 9 rely on earlier results that use much simpler topological tools than those involved in Theorem 3 and 4. In particular, the proofs of Theorems 2, 5, 7, 8, and 9 are independent from Sections 2 and 3. On the other hand, the proofs of Theorems 3 and 4 heavily depend on the topological results from the previous sections.

4.1. Proof of Theorem 2. Let $d \geqslant 2, m \geqslant 2, n \geqslant 2$ and $c \geqslant d$ be integers such that $m \geqslant n(c-d)+d$. Since the measures $\mu_{1}, \ldots, \mu_{m}$ are positive, finite, and absolutely continuous with respect to the standard Lebesgue measure, the interiors of their supports are nonempty. For each $1 \leqslant j \leqslant m$, choose a point $v_{j} \in \operatorname{int}\left(\operatorname{supp}\left(\mu_{j}\right)\right)$ in the interior of the support of the measure $\mu_{j}$. Set $V:=$ $\left\{v_{1}, \ldots, v_{m}\right\}$. Perturb the points $v_{1}, \ldots, v_{m}$ if necessary, so that they are in general position, that is no $d+1$ of them lie in the same affine hyperplane. The set $P:=\operatorname{conv}(V)$ is a $d$-dimensional simplicial polytope in $\mathbb{R}^{d}$. Choose any $(d-2)$ dimensional face $F$ of the polytope $P$. Since $P$ is simplicial the face $F$ is a simplex and so has $d-1$ vertices that belong to $V$.

First, we look for an affine hyperplane $H$ in $\mathbb{R}^{d}$ with the properties that:

$-F \subseteq H$,

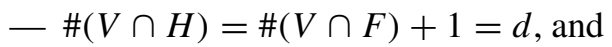

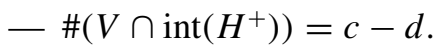

The hyperplane $H$ cuts $\mathbb{R}^{d}$ into two half-spaces closed half-spaces $H^{+}$and $H^{-}$ such that $\mathrm{H}^{+}$has positive measure with respect to at least $c$ of the measures $\mu_{1}, \ldots, \mu_{m}$, because it intersects interiors of supports of at least $c$ measures. 
Such a hyperplane exists. Indeed, since $F$ is a face of $P$ there exists a supporting hyperplane $H^{\prime}$ for $F$, that is a hyperplane that contains the face $F$ and one of its closed half-spaces contains $P$, see Figure 3(a). Rotate the hyperplane $H^{\prime}$ around the $(d-2)$-dimensional subspace spanned by $F$ to get the hyperplane $H$ such that there are exactly $c-d$ points of $V$ in $\operatorname{int}\left(H^{+}\right)$, and furthermore there is one additional point from $V \backslash F$ on $H$. Since the affine span of $F$ is a hyperplane in $H$ the additional point, say $w$, lies in the relative interior of one of the half-hyperplanes of $H$ determined by $F$. Denote the half-hyperplanes of $H$ determined by $F$ with $K_{0}$ and $K_{m-c+1}$ such that $w \in \operatorname{relint}\left(K_{0}\right)$. In particular, $K_{0} \cup K_{m-c+1}=H$.

The set $V^{-}:=V \cap H^{-}$is of cardinality $m-c$. Consider all half-hyperplanes whose boundary is the affine span of $F$ and contains a point of $V^{-}$in its relative interior. Since the set $V$ is in general position, there are exactly $m-c$ such halfhyperplanes. Label them $K_{1}, \ldots, K_{m-c}$ in order, starting from the half-hyperplane that forms the smallest angle with the half-hyperplane $K_{0}$, as illustrated in Figure 3(c). The affine span of $F$ and the half-hyperplanes

$$
K_{0}, K_{c-d}, K_{2(c-d)}, \ldots, K_{(n-2)(c-d)}, K_{m-c+1}
$$

define an $n$-fan whose every region intersects interiors of the supports of at least $c$ of the measures $\mu_{1}, \ldots, \mu_{m}$. Indeed, the region defined by

- $H^{+}$, or equivalently by $K_{m-c+1}$ and $K_{0}$ contains exactly $c$ points from $V$,

$-K_{0}$ and $K_{c-d}$ contains exactly $d-1+c-d+1=c$ points from $V$,

$-K_{c-d}$ and $K_{2(c-d)}$ contains exactly $d-1+c-d+1=c$ points from $V$,

- $K_{(n-2)(c-d)}$ and $K_{m-c+1}$ contains $d-1+m-c-(n-2)(c-d)+1=$ $(m-n c+n d-d)+c \geqslant c$ points from $V$.

An example for $d=2, n=5$ and $c=4$ is shown in Figure 3(d).

Thus, we constructed a convex partition of $\mathbb{R}^{d}$ such that each piece of the partition has a positive measure with respect to at least $c$ of the measures $\mu_{1}$, $\ldots, \mu_{m}$. This concludes the proof of the theorem.

REMARK 24. As a consequence of the previous proof, there is a convex partition $\left(C_{1}, \ldots, C_{n}\right)$ of $\mathbb{R}^{d}$, such that each piece $C_{i}$ has positive measure with respect to at least $c$ of the measures $\mu_{1}, \ldots, \mu_{m}$, and additionally all pieces $C_{1}, \ldots, C_{n}$ have positive measure with respect to $d-1$ measures $\mu_{j_{1}}, \ldots, \mu_{j_{d-1}}$, where $F=$ $\operatorname{conv}\left\{v_{j_{1}}, \ldots, v_{j_{d-1}}\right\}$ and $v_{j_{k}} \in \operatorname{relint}\left(\operatorname{supp}\left(\mu_{j_{k}}\right)\right)$, for every $1 \leqslant k \leqslant d-1$. In contrast to the statement of Theorem 3 , we cannot guarantee an equipartition, and we cannot choose which measure will be contained in each piece. 


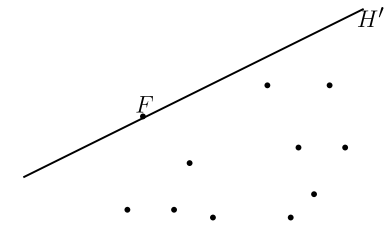

(a) The face $F$ and the hyperplane $H^{\prime}$.

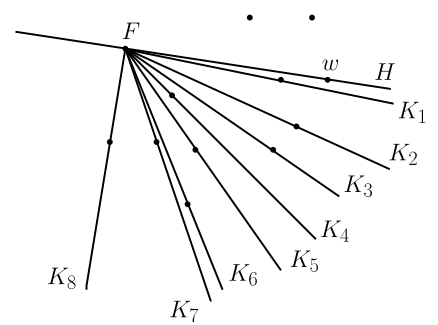

(c) Labeling of the half-hyperplanes in halfspace $H^{-}$.

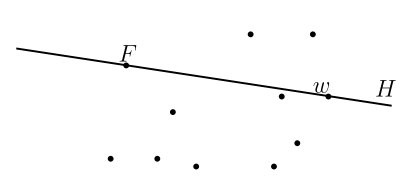

(b) The face $F$, the point $w$ and the final position of the hyperplane $H^{\prime}=H$.

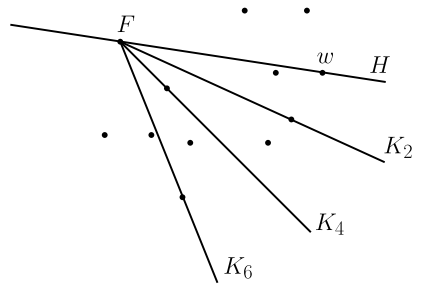

(d) A 5-fan that partitions $\mathbb{R}^{2}$ into convex pieces so that each piece has positive measure with respect to at least 4 measures.

Figure 3. An example of a fan partition of $\mathbb{R}^{2}$ for $n=5$ and $c=4$.

4.2. Proof of Theorem 3. Let $d \geqslant 2, m \geqslant 2$, and $c \geqslant 2$ be integers, and let $n \geqslant 2$ be a prime power. Under the assumptions of the theorem on $m$, Theorem 17 yields the nonexistence of an $\mathfrak{S}_{n}$-equivariant map

$$
\operatorname{EMP}\left(\mu_{m}, p\right) \longrightarrow \bigcup \mathcal{A}(m, n, c)
$$

Consequently, Theorem 10 implies that for every collection of $m$ measures $\mu_{1}$, $\ldots, \mu_{m}$ in $\mathbb{R}^{d}$ there exists a convex partition $\left(C_{1}, \ldots, C_{n}\right)$ of $\mathbb{R}^{d}$ with the property that each of the subsets $C_{i}$ has positive measure with respect to at least $c$ of the measures $\mu_{1}, \ldots, \mu_{m}$. In other words,

$$
\#\left\{j: 1 \leqslant j \leqslant m, \mu_{j}\left(C_{i}\right)>0\right\} \geqslant c
$$

for every $1 \leqslant i \leqslant n$.

REMARK 25. In order to prove the nonexistence of the $G$-equivariant map $f: \operatorname{EMP}\left(\mu_{m}, n\right) \longrightarrow \bigcup \mathcal{A}$, one could directly try to show that there is no $G$-equivariant map $\operatorname{Conf}\left(\mathbb{R}^{d}, n\right) \longrightarrow \bigcup \mathcal{A}=\operatorname{colim}_{P(\mathcal{A})} \mathcal{C}$. However, since the 
dimension of the order complex of $P(\mathcal{A})$ is

$$
\operatorname{dim}(\Delta(P(\mathcal{A})))=n c-n-c,
$$

this method proves Theorem 3 only for $c \leqslant d$, which follows directly from the result of Soberón [16].

4.3. Proof of Theorem 4. Let $d \geqslant 2$ and $c \geqslant 2$ be integers, let $n \geqslant 2$ be a prime power and let $m \geqslant 2$ be an integer that satisfies the conditions of the theorem. Theorem 23 yields the nonexistence of an $\mathfrak{S}_{n}$-equivariant map

$$
\operatorname{EMP}(\mu, p) \longrightarrow \bigcup \tilde{\mathcal{A}}(m, n, c)
$$

and Theorem 11 implies that for every collection of $m$ measures $\mu_{1}, \ldots, \mu_{m}$ in $\mathbb{R}^{d}$ there exists a convex partition $\left(C_{1}, \ldots, C_{n}\right)$ of $\mathbb{R}^{d}$ with the property that each of the subsets $C_{i}$ has positive measure with respect to at least $c$ of the measures $\mu_{1}, \ldots, \mu_{m}$. In other words,

$$
\#\left\{j: 1 \leqslant j \leqslant m, \mu_{j}\left(C_{i}\right)>0\right\} \geqslant c
$$

for every $1 \leqslant i \leqslant n$.

4.4. The first proof of Theorem 5. Let $d \geqslant 2, n \geqslant 2$ and $c \geqslant d$ be integers, and set $m=n(c-d)+d$. Consider $m$ measures $\mu_{1}, \ldots, \mu_{m}$ on $\mathbb{R}^{d}$ which are positive, finite and absolutely continuous with respect to the standard Lebesgue measure. Thus, the interiors of their supports are nonempty. For each $d \leqslant j \leqslant m$, choose a point $v_{j} \in \operatorname{int}\left(\operatorname{supp}\left(\mu_{j}\right)\right)$ in the interior of the support of the measure $\mu_{j}$. Now consider the set $V:=\left\{v_{d}, \ldots, v_{m}\right\}$ as a point measure.

The result of Soberón [16] applied to the collection of measures $\mu_{1}, \ldots, \mu_{d-1}$, $V$ guaranties the existence of a convex partition $\left(C_{1}, \ldots, C_{n}\right)$ of $\mathbb{R}^{d}$ that equiparts $\mu_{1}, \ldots, \mu_{d-1}$ and in addition the point measure $V$. For the point measure $V$ it means that

$$
\#\left(V \cap C_{i}\right) \geqslant\left\lceil\frac{m-d+1}{n}\right\rceil \geqslant\left\lceil\frac{n(c-d)+d-d+1}{n}\right\rceil=c-d+1,
$$

for every $1 \leqslant i \leqslant n$. Consequently,

$$
\#\left\{j: 1 \leqslant j \leqslant m, \mu_{j}\left(C_{i}\right)>0\right\} \geqslant c
$$

for all $1 \leqslant i \leqslant n$. 


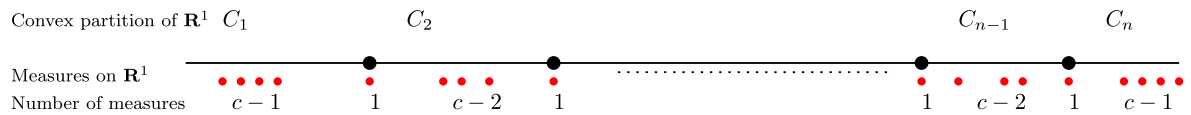

Figure 4. Optimality of the bound in Theorem 7 in the case $d=1$.

4.5. The second proof of Theorem 5. Let $d \geqslant 2, n \geqslant 2$ and $c \geqslant d$ be integers, and let $m=n(c-d)+d$. Consider $m$ measures $\mu_{1}, \ldots, \mu_{m}$ on $\mathbb{R}^{d}$ which are positive, finite and absolutely continuous with respect to the standard Lebesgue measure. We introduce a new measure $v$ by

$$
\nu(A)=\sum_{i=d}^{m} \frac{\mu_{i}(A)}{\mu_{i}\left(\mathbb{R}^{d}\right)},
$$

for $A \subseteq \mathbb{R}^{d}$ a measurable set. Then $v\left(\mathbb{R}^{d}\right)=m-(d-1)=n(c-d)+1$, and each of the measures $\mu_{d}, \ldots, \mu_{m}$ can contribute at most 1 to $v(A)$ for any $A \subseteq \mathbb{R}^{d}$. Therefore, if $v(A)>k$ for some nonnegative integer $k$, then $A$ must have positive size in at least $k+1$ of the measures from $\mu_{d}, \ldots, \mu_{m}$.

Again, the result of Soberón [16] applied to the collection of measures $\mu_{1}, \ldots$, $\mu_{d-1}, v$ guaranties the existence of a convex partition $\left(C_{1}, \ldots, C_{n}\right)$ of $\mathbb{R}^{d}$ that equiparts measures $\mu_{1}, \ldots, \mu_{d-1}$ as well as the measure $\nu$. In particular,

$$
v\left(C_{i}\right)=\frac{n(c-d)+1}{n}>c-d \geqslant 0
$$

for every $1 \leqslant i \leqslant n$. Therefore, each of the pieces $C_{i}$ of the partition has positive size in at least $c-d+1$ measures among $\mu_{d}, \ldots, \mu_{m}$. Having in addition exactly $1 / n$ fraction of each of the measures $\mu_{1}, \ldots, \mu_{d-1}$ each convex piece $C_{i}$ of the partition has positive size in at least $c$ measures among $\mu_{1}, \ldots, \mu_{m}$.

4.6. Proof of Theorem 7. First, we prove that $m=n(c-d)+d$ is the optimal bound in Theorem 7 for $d=1$. It suffices to consider $n(c-1)+1$ measures on $\mathbb{R}$, each concentrated near a point, so that the supports are pairwise disjoint. Each convex partition is given by a family of intervals whose interiors are pairwise disjoint. Thus, consecutive intervals can share the support of at most one measure. A careful counting, as illustrated in Figure 4, shows that $n(c-1)+1$ measures are needed for each interval to intersect the support of $c$ measures.

For the case $d \geqslant 2$ we use the following construction illustrated in Figure 5. Let $e_{1}, \ldots, e_{d}$ denote the vector of the standard basis, and let $\ell$ be the line $e_{d}+$ $\operatorname{span}\left(e_{d-1}\right)$. We place $m-d+1$ measure $\mu_{d}, \ldots, \mu_{m}$ concentrated in points of $\ell$, as in the case of dimension 1 . Now consider a regular simplex in the affine 


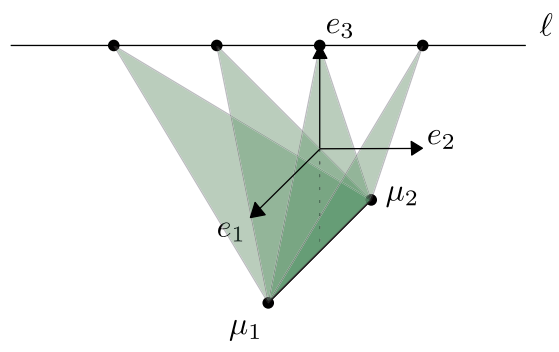

Figure 5. Optimality of the bound in Theorem 7 in the case $d \geqslant 2$.

subspace $-e_{d}+\operatorname{span}\left(e_{1}, \ldots, e_{d-2}\right)$, of dimension $d-2$, centered around $-e_{d}$. Place $d-1$ measures $\mu_{1}, \ldots, \mu_{d-1}$, each concentrated near a vertex of the simplex we just constructed. We ask from our partition into convex subsets to equiparts the measures $\mu_{1}, \ldots, \mu_{d-1}$. Thus, each piece of the convex partition has positive size in $\mu_{1}, \ldots, \mu_{d-1}$. In order for the interior of their convex hulls to be disjoint, they have to intersect the remaining measures $\mu_{d}, \ldots, \mu_{m}$ as in the case of dimension 1 . Since they must each intersect at least $c-(d-1)$ measure on the line $\ell$, and $\ell$ has $m-(d-1)$ measures, the case of dimension one implies that

$$
m-(d-1) \geqslant n(c-(d-1)-1)+1 \Longleftrightarrow m \geqslant n(c-d)+d .
$$

This concludes the proof of the theorem.

4.7. Proof of Theorem 8. For the proof of the theorem we first derive the following version of the pigeonhole principle.

Claim 26. Let $m \geqslant 1$ and $1 \leqslant r \leqslant m$ be integers, and let $0 \leqslant x_{1}, \ldots, x_{m} \leqslant 1$ and $0 \leqslant \varepsilon \leqslant 1$ be real numbers. If

$$
x_{1}+\cdots+x_{m} \geqslant r-1+\varepsilon(m-r+1),
$$

then there exist at least $t$ indices $1 \leqslant i_{1}<\cdots<i_{t} \leqslant m$ such that

$$
\min \left\{x_{i_{1}}, \ldots, x_{i_{r}}\right\} \geqslant \varepsilon .
$$

Proof. Suppose that the claim does not hold. Without lost of generality we can assume that

$$
0 \leqslant x_{m} \leqslant \cdots \leqslant x_{r} \leqslant x_{r-1} \leqslant \cdots \leqslant x_{1} \leqslant 1
$$

Then

$$
x_{m} \leqslant \cdots \leqslant x_{r}<\varepsilon
$$


Consequently,

$$
x_{1}+\cdots+x_{m}=\left(x_{1}+\cdots+x_{r-1}\right)+\left(x_{r}+\cdots+x_{m}\right)<r-1+\varepsilon(m-r+1) \text {. }
$$

We reached a contradiction with the assumption (18).

Now we proceed with the proof of Theorem 8 . Let $d \geqslant 2, n \geqslant 2$ and $c \geqslant d$ be integers, and let $m=n(c-d)+d$. Consider $m$ measures $\mu_{1}, \ldots, \mu_{m}$ on $\mathbb{R}^{d}$ which are positive, finite and absolutely continuous with respect to the standard Lebesgue measure. We partition our set of $m$ measures into $d$ subsets $I_{1}, \ldots, I_{d}$ such that

$-c=\sum_{k=1}^{d} r_{k} \geqslant d$ for some positive integers $r_{1}, \ldots, r_{d}$, and

- \#I $I_{k}=m_{k}=n\left(r_{k}-1\right)+1$ for all $1 \leqslant k \leqslant d$.

For each $1 \leqslant k \leqslant d$ we define the measure $v_{k}$ on $\mathbb{R}^{d}$ by

$$
v_{k}(A)=\sum_{\mu \in I_{k}} \frac{\mu(A)}{\mu\left(\mathbb{R}^{d}\right)},
$$

where $A \subseteq \mathbb{R}^{d}$ is a measurable set. Consequently, $v_{k}\left(\mathbb{R}^{d}\right)=\# I_{k}=n\left(r_{k}-1\right)+1$.

Using the result of Soberón [16] applied to the collection of measures $v_{1}, \ldots$, $v_{d}$ we get a convex partition $\left(C_{1}, \ldots, C_{n}\right)$ of $\mathbb{R}^{d}$ with the property that

$$
v_{k}\left(C_{i}\right)=\frac{v_{k}\left(\mathbb{R}^{d}\right)}{n}=r_{k}-1+\frac{1}{n}
$$

for every $1 \leqslant k \leqslant d$ and every $1 \leqslant i \leqslant n$.

Now fix $k$ and $i$, and consider $m_{k}$ real numbers $\mu\left(C_{i}\right) / \mu\left(\mathbb{R}^{d}\right), \mu \in I_{k}$, in the interval $[0,1]$. Since

$$
\sum_{\mu \in I_{k}} \frac{\mu\left(C_{i}\right)}{\mu\left(\mathbb{R}^{d}\right)}=v_{k}\left(C_{i}\right)=r_{k}-1+\frac{1}{n},
$$

we can apply Claim 26 to get at least $r_{k}$ numbers out of $\mu\left(C_{i}\right) / \mu\left(\mathbb{R}^{d}\right), \mu \in I_{k}$, greater than or equal to $\varepsilon_{k}=1 /\left(\left(n\left(m_{k}-r_{k}+1\right)\right)\right.$. Thus, there are at least $r_{k}$ measures $\mu$ in the set $I_{k}$ such that

$$
\mu\left(C_{i}\right) \geqslant \varepsilon_{k} \mu\left(\mathbb{R}^{d}\right)=\frac{1}{n\left(m_{k}-r_{k}+1\right)} \mu\left(\mathbb{R}^{d}\right),
$$

where $1 \leqslant k \leqslant d$. Now, we choose each $r_{k}$ to be either $\lfloor c / d\rfloor$ or $\lceil c / d\rceil$ in such a way that $\sum_{k=1}^{d} r_{k}=c$. Consequently, we obtain a uniform lower bound for each fraction $\varepsilon_{k}$ that we were seeking for.

Hence, each of the subsets $C_{1}, \ldots, C_{n}$ has at least $\varepsilon$ fraction in at least $c$ of the measures $\mu_{1}, \ldots, \mu_{m}$, and the proof of the theorem is concluded. 
4.8. Proof of Theorem 9. The proof of the theorem proceeds along the lines of the proof of Theorem 8 presented in Section 4.7. Let $d \geqslant 2, m \geqslant 2, n \geqslant 2$, and $c \geqslant 2 d$ be integers, and let $0<\alpha<1 / n$ be a real number. Assume that

$$
m \geqslant(c-d)\left(\frac{1-\alpha}{1 / n-\alpha}\right)+d-1 .
$$

Let $r_{1}, \ldots, r_{d}$ be integers such that $r_{k} \geqslant 2$ for all $1 \leqslant k \leqslant d$, and let $r_{1}+\cdots+$ $r_{d}=c$. For each $1 \leqslant k \leqslant d$ we set

$$
m_{k}=\left\lceil\left(r_{k}-1\right)\left(\frac{1-\alpha}{1 / n-\alpha}\right)\right\rceil>0 .
$$

Then $m_{k}<\left(r_{k}-1\right)((1-\alpha) /(1 / n-\alpha))+1$ for $1 \leqslant k \leqslant d$, and consequently

$$
m_{1}+\cdots+m_{d}<(c-d)\left(\frac{1-\alpha}{1 / n-\alpha}\right)+d \leqslant m+1 .
$$

Since $m, m_{1}, \ldots, m_{d}$ are all integers, the previous inequality implies that

$$
m_{1}+\cdots+m_{d} \leqslant m \text {. }
$$

Now, out of the set of $m$ measures $\left\{\mu_{1}, \ldots, \mu_{m}\right\}$ we can choose $d$ nonempty disjoint subsets of measures $I_{1}, \ldots, I_{d}$ such that \# $I_{k}=m_{k}, 1 \leqslant k \leqslant d$. For each $1 \leqslant k \leqslant d$ we define, as before, the measure $v_{k}$ on $\mathbb{R}^{d}$ by

$$
v_{k}(A)=\sum_{\mu \in I_{k}} \frac{\mu(A)}{\mu\left(\mathbb{R}^{d}\right)},
$$

where $A \subseteq \mathbb{R}^{d}$ is a measurable set. Hence,

$$
v_{k}\left(\mathbb{R}^{d}\right)=\# I_{k}=m_{k}=\left\lceil\left(r_{k}-1\right)\left(\frac{1-\alpha}{1 / n-\alpha}\right)\right\rceil \geqslant\left(r_{k}-1\right)\left(\frac{1-\alpha}{1 / n-\alpha}\right) .
$$

The inequality

$$
m_{k} \geqslant\left(r_{k}-1\right)\left(\frac{1-\alpha}{1 / n-\alpha}\right)
$$

can be rearranged into

$$
\frac{m_{k}}{n} \geqslant r_{k}-1+\alpha\left(m_{k}-r_{k}+1\right)
$$

The result of Soberón [16] applied on the collection of measures $v_{1}, \ldots, v_{d}$ gives a convex partition $\left(C_{1}, \ldots, C_{n}\right)$ of $\mathbb{R}^{d}$ with the property that

$$
v_{k}\left(C_{i}\right)=\frac{v_{k}\left(\mathbb{R}^{d}\right)}{n}=\frac{m_{k}}{n} \geqslant r_{k}-1+\alpha\left(m_{k}-r_{k}+1\right) .
$$


for every $1 \leqslant k \leqslant d$ and every $1 \leqslant i \leqslant n$. Now, fix any $1 \leqslant i \leqslant n$ and $1 \leqslant k \leqslant d$. Consider the $m_{k}$ numbers of the form $\mu\left(C_{i}\right) / \mu\left(\mathbb{R}^{d}\right)$ for $\mu \in I_{k}$. From Claim 26, it follows that at least $r_{k}$ of those numbers are at least $\alpha$. If we repeat this for every $k$, we obtain that $C_{i}$ has a fraction $\alpha$ of at least $r_{1}+\cdots+r_{k}=c$ measures, as desired.

If the number $(1-\alpha) /((1 / n)-\alpha)$ is an integer, then we set $m_{k}=\left(r_{k}-1\right)$ $(1-\alpha) /(1 / n-\alpha)$. Consequently, we only require the bound

$$
m \geqslant(c-d)\left(\frac{1-\alpha}{1 / n-\alpha}\right)
$$

on the number of measures to derive the theorem.

REMARK 27. One can observe that the extra ' $+(d-1)$ ' on the bound of $m$ is not always needed, but the precise value would then depend on a careful choice of the parameters $r_{1}, \ldots, r_{d}$, which would require a case-by-case analysis. Some cases yield very clean bounds. For example, if $\alpha=1 /(2 n-1)$, we are getting many measures that have more than half of what would be the optimal bound. Indeed, for $m=2 n(c-d)$ measures in $\mathbb{R}^{d}$, there exists a partition of $\mathbb{R}^{d}$ into $n$ convex parts such that each part has at least $1 /(2 n-1)$ fraction of at least of $c$ measures.

\section{Acknowledgements}

We are grateful to Jonathan Kliem, Johanna K. Steinmeyer and Roman Karasev for many useful observations and suggestions. The research by Pavle V. M. Blagojević leading to these results has received funding from DFG via the Collaborative Research Center TRR 109 'Discretization in Geometry and Dynamics', and the grant ON 174024 of the Serbian Ministry of Education and Science. The research by Nevena Palic leading to these results has received funding from DFG via the Berlin Mathematical School. The research by Günter M. Ziegler leading to these results has received funding from DFG via the Collaborative Research Center TRR 109 'Discretization in Geometry and Dynamics'. This material is based on the work supported by the National Science Foundation under Grant No. DMS-1440140 while Blagojević, Palić, and Ziegler were in residence at the Mathematical Sciences Research Institute in Berkeley CA, during the Fall of 2017. The research of Pablo Soberón leading to these results has received funding from the National Science Foundation under Grant No. DMS-1851420. We acknowledge the support by the German Research Foundation and the OpenAccess Publication Fund of Freie Universität Berlin. 


\section{Conflict of Interest: None.}

\section{References}

[1] A. Adem and J. R. Milgram, Cohomology of Finite Groups, 2nd edn, Grundlehren der Mathematischen Wissenschaften, vol. 309 (Springer, Berlin, 2004).

[2] I. Bárány and J. Matoušek, 'Simultaneous partitions of measures by k-fans', Discrete Comput. Geom. 25 (2001), 317-334.

[3] P. V. M. Blagojević, F. Frick, A. Haase and G. M. Ziegler, 'Topology of the GrünbaumHadwiger-Ramos hyperplane mass partition problem', Trans. Amer. Math. Soc. 370(10) (2018), 6795-6824.

[4] P. V. M. Blagojević, W. Lück and G. M. Ziegler, 'Equivariant topology of configuration spaces', J. Topol. 8 (2015), 414-456.

[5] P. V. M. Blagojević, G. Rote, J. K. Steinmeyer and G. M. Ziegler, 'Convex equipartitions of colored point sets', Discrete Comput. Geom. 61 (2019), 355-363.

[6] P. V. M. Blagojević and G. M. Ziegler, 'Convex equipartitions via equivariant obstruction theory', Israel J. Math. 200 (2014), 49-77.

[7] A. K. Bousfield and D. M. Kan, Homotopy Limits, Completions and Localizations, Lecture Notes in Mathematics, 304 (Springer, Berlin, 1972), Heidelberg, New York.

[8] F. R. Cohen, 'The homology of $\mathcal{C}_{n+1}$-spaces, $n \geqslant 0$ ', in The Homology of Iterated Loop Spaces, Vol. 533 (eds. F. R. Cohen, T. J. Lada and J. Peter May) Lecture Notes in Mathematics (Springer, Berlin, 1976), 207-351. Heidelberg, New York.

[9] B. Grünbaum, 'Partitions of mass-distributions and of convex bodies by hyperplanes', Pacific J. Math. 10 (1960), 1257-1261.

[10] H. Hadwiger, 'Simultane Vierteilung zweier Körper', Arch. Math. 17 (1966), 274-278.

[11] A. Hatcher, Algebraic Topology, (Cambridge University Press, Cambridge, 2002).

[12] A. F. Holmsen, J. Kynčl and C. Valculescu, 'Near equipartitions of colored point sets', Comput. Geom. 65 (2017), 35-42.

[13] W. Y. Hsiang, Cohomology Theory of Topological Transformation Groups (Springer, New York-Heidelberg, 1975), Ergebnisse der Mathematik und ihrer Grenzgebiete, Band 85.

[14] E. León and G. M. Ziegler, 'Spaces of convex n-partitions', in New Trends in Intuitive Geometry, (eds. G. Ambrus, I. Bárány, K. J. Böröczky, G. Fejes Tóth and J. Pach) Bolyai Society Mathematical Studies, 27 (Springer, Berlin Heidelberg, 2018), 279-306.

[15] E. A. Ramos, 'Equipartition of mass distributions by hyperplanes', Discrete Comput. Geom. 15 (1996), 147-167.

[16] P. Soberón, 'Balanced convex partitions of measures in $\mathbb{R}^{d}$, Mathematika 58 (2012), 71-76.

[17] S. Sundaram and V. Welker, 'Group actions on arrangements of linear subspaces and applications to configuration spaces', Trans. Amer. Math. Soc. 349 (1997), 1389-1420.

[18] A. Y. Volovikov, 'On the index of G-spaces', Mat. Sb. 191 (2000), 3-22.

[19] V. Welker, G. M. Ziegler and R. T. Živaljević, 'Homotopy colimits-comparison lemmas for combinatorial applications', J. Reine Angew. Math. 509 (1999), 117-149. 